\title{
Direct Numerical Simulation of Supersonic Turbulent Flows around a Tandem Expansion-Compression Corner
}

\author{
Jian Fang, ${ }^{1,2}$ Yufeng Yao, ${ }^{3}$ Alexander A. Zheltovodov, ${ }^{4}$ Zhaorui Li, ${ }^{5}$ and Lipeng Lu ${ }^{1, \text { a) }}$ \\ ${ }^{1}$ National Key Laboratory of Science and Technology on Aero-Engine Aero-Thermodynamics, School of Energy and \\ Power Engineering, Beihang University, Beijing 100191, China \\ ${ }^{2}$ Computer Science and Engineering Department, STFC Daresbury Laboratory, Warrington WA4 4AD, United \\ Kingdom \\ ${ }^{3}$ Faculty of Environment and Technology, Department of Engineering Design and Mathematics, University of the West \\ of England, Bristol BS16 1QY, United Kingdom \\ ${ }^{4}$ Khristianovich Institute of Theoretical and Applied Mechanics, Siberian Branch of Russian Academy of Science, \\ Novosibirsk 630090, Russia \\ ${ }^{5}$ School of Engineering and Computing Sciences, Texas A\&M University-Corpus Christi, 6300 Ocean Drive, Corpus \\ Christi, TX 78412, USA
}

The $M=2.9$ supersonic turbulent flows over a tandem expansion-compression corner configuration with a sharp deflection angle of $25^{\circ}$ at three Reynolds numbers $\operatorname{Re}_{\delta}=20000,40000$ and 80000 were studied by using direct numerical simulation (DNS). The flow statistics were validated against available experimental measurements and other numerical predictions. The flow structures and turbulence statistics were detailed visualized and analysed for the $\operatorname{Re}_{\delta}=40000$ case, especially in the interaction region where flow separation and reattachment occurred. It was found that during the expansion process the boundary layer exhibited a characteristic two-layer structure also discovered in previous experimental studies, and the turbulence evolved differently within these two layers. In the outer layer the turbulence was consistently suppressed along the ramp to a large extent, while in the inner layer it was suppressed only in a small region around the expansion corner, and the near-wall quasi-streamwise vortices were well preserved. Flow patterns near the reattachment line have shown the existence of the Görtler-type vortices, which would largely amplify turbulence fluctuations in the near-wall region, thus promoting the regeneration of wall turbulence that in turn contributed to the redevelopment of a downstream turbulent boundary layer. The Reynolds number effects and the characteristics of coherent structures were also discussed. With the increase of the Reynolds number, the separation bubble size decreased, but the pattern and the characteristic size of wall streamlines near the reattachment line were preserved.

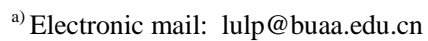




\section{INTRODUCTION}

Shock-wave/turbulent boundary layer interactions (SWTBLI) are prevalent phenomena in high-speed flights. The resultant strong adverse pressure gradient (APG) caused by SWTBLI will induce large flow separation, high wall heat flux and strong pressure fluctuation, along with other complex physical phenomena ${ }^{1-4}$. Therefore, SWTBLI significantly affects the aerodynamic and thermodynamic loads of the flying vehicles and also the performance of their propulsion systems. Among various kinds of SWTBLI, the supersonic flow over a tandem expansion-compression corner has been tested in wind tunnels as a model representing a class of expansion-compression flows, such as fore-body, intake, combustion chamber and nozzle of high-speed flying vehicles. In an expansion-compression corner configuration shown in FIG. 1, the supersonic flow is accelerated and turned along with a convex streamline curvature through the expansion fan at the expansion corner (EC), then compressed by the shock-wave around the compression corner $(\mathrm{CC})^{2}$. The boundary layer in the compression corner region can be either attached or separated, depending on the shock-wave strength. This kind of flow is essentially in a non-equilibrium state, due to the strong interactions among turbulence, expansion-wave and shock-wave.

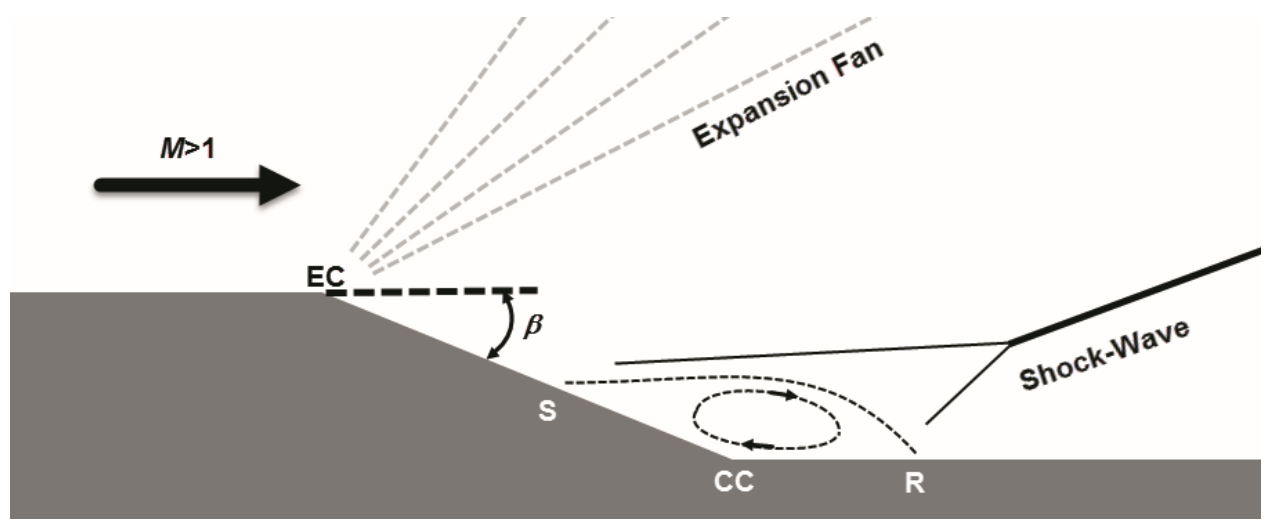

FIG. 1. A sketch of an expansion-compression corner configuration: ' $\mathrm{S}$ ' - separation point; 'R' - reattachment point; 'EC' - expansion corner; 'CC' - compression corner, ' $\beta$ ' - defection angle.

Despite of the importance of the expansion-compression corner flow in high-speed vehicles, it has not been studied as much as supersonic compression corner flow or impinging shock-wave/boundary layer interactions ${ }^{2-4}$. Early researches mainly focused on the pressure gradient and the curvature effects of the expansion part of the configuration, including the increase of the boundary layer thickness, the reduction of turbulence intensity and heat transfer, the stabilization and the relaminarization of the boundary layer during the expansion process, etc. ${ }^{5-16} \mathrm{~A}$ comprehensive review of researches on the supersonic flow passing an isolated expansion corner was presented by Knight et al. ${ }^{2}$

The suppression of turbulence during the expansion process ${ }^{6,10,12-17}$ is a significant characteristic in expansioncompression corner flows, and could cause the re-laminarization of the boundary layer when the flow undergoes a strong expansion process. ${ }^{11,12,17}$ The reason for the turbulence being suppressed could be related to the combined effects of the favourable pressure gradient (FPG), the convex curvature of streamlines and the bulk dilatation (see the review of Knight et 
al. $^{2}$ ). Early researches into the FPG and bulk dilatation effects were conducted for turbulent boundary layers with simple geometries, such as the suppression of Reynolds stresses and turbulence transport due to the FPG and bulk dilatation in a supersonic boundary layer studied by Morkovin ${ }^{6}$ and Lewis et al. ${ }^{10}$ The streamline curvature effect was also discussed in previous researches, most of them ${ }^{18-20}$ being focused on the boundary layer with constant curvature. The relation between the boundary layer parameters and the curvature was the primary interest of those studies.

Sternberg ${ }^{5}$ proposed the first model for the boundary layer structure in the supersonic flow around a cone-cylinder configuration on the basis of experimental observations. According to this model, the initial turbulent boundary layer on the cone surface becomes laminar close to the wall immediately behind the junction of the cone with the cylinder. However, transition of this laminar boundary layer to turbulence happens again on the cylindrical surface at some distance downstream of the surface discontinuity. Consequently, a specific two-layer structure develops behind the expansion corner because the initial boundary layer from the cone remains and develops as a turbulent shear layer above the appeared near-wall laminar boundary layer. Based on the experimental observations in a specially designed curved channel, Gillis et al. ${ }^{20}$ also proposed a two-layer model for the boundary layer flow during the expansion and the recovery processes, which includes the near-wall active layer where turbulence is anisotropic and still in production, and the outer layer containing nearly isotropic 'debris' inherited from the upstream thick boundary layer. The two-layer model was adopted by Zakkay et al. ${ }^{14}$ to treat the boundary layer and surface heat transfer past a sharp expansion corner.

The considered two-layer model was confirmed by later detailed experimental investigations, for example, by Dussauge and Gaviglio ${ }^{12}$ as well as by Goldfeld et al. ${ }^{17}$. According to these experiments, the partial re-laminarization of the boundary layer downstream of the expansion corner at a sufficient favourable pressure gradient is possible. The near-wall part of the boundary layer becomes laminar and is well described by the theoretical Blasius mean velocity profile. ${ }^{17}$ The thickness of this part of the boundary layer is significantly larger than the typical values of the laminar sub-layer (also called linear sub-layer) of the turbulent boundary layer. At the same time, the external part of the boundary layer does not have a clear logarithmic section, but there is a typical wake component of a turbulent velocity profile. The action of a strong FPG leads to a significant decrease in the levels of velocity fluctuations ${ }^{12}$ and mass-flow fluctuations ${ }^{17}$ immediately behind the expansion corner. The profile of the mass-flow fluctuation in the re-laminarization region corresponds to the two-layer boundary layer structure with two peaks. One of the peaks is at the boundary-layer edge and increases insignificantly downstream. The other peak is in the near-wall part of the boundary layer immediately behind the corner and rapidly increases downstream. A similar tendency of the increase of near-wall velocity fluctuation peaks was also observed. ${ }^{12}$ 
Zheltovodov et al. ${ }^{21-24}$ conducted systematic and comprehensive measurements on the expansion-compression corner flow problems in early 1990s and derived three basic characteristics of the flow regimes: (1) the attached flow at a small angle $\beta$, (2) the appearance of a local separation zone in the $\mathrm{CC}$ region with a free separation point at a moderate angle $\beta$, and (3) the formation of a large-scale separated flow with a fixed separation point at a large angle $\beta$. The relationship between the characteristic length of flow separation and the incoming boundary layer thickness and Mach number was revealed by Zheltovodov and Schülein ${ }^{22}$ as well as by Zheltovodov et al. ${ }^{24}$ The appearance of Görtler-type vortices were also reported by Zheltovodov et al. ${ }^{21}$ with the help of the surface oil flow pattern visualization.

Later, Borisov et al. ${ }^{25}$, Zheltovodov et al. ${ }^{26}$, Zheltovodov and Horstman ${ }^{27}$, as well as Horstman and Zheltovodov ${ }^{28}$ conducted joint researches combining numerical simulations and experimental measurements. It was found that ReynoldsAveraged Navier-Stokes (RANS) method, with different turbulence models, could give fairly good predictions of general flowfield features, except for the non-equilibrium region of the flow, where the separation and reattachment points were overpredicted. Moreover, the prediction of the surface heat transfer coefficient was totally misleading (see the survey by Zheltovodov ${ }^{3}$ ). Therefore, the credibility of applying RANS-based approaches for in-depth researches of this type of highspeed flows is quite limited, due to the incapability of turbulence models in predicting complex non-equilibrium turbulent flows. Hence, more accurate and high-fidelity numerical methods such as direct numerical simulation (DNS) and large-eddy simulation (LES) would be necessary to investigate this kind of flow phenomena.

In DNS and/or LES, small-scale turbulence fluctuations can be either directly resolved or modelled up to the sub-grid scale (SGS) level. Therefore, they can provide detailed flow information and accurate predictions. Nowadays, DNS and LES are playing very important roles in turbulence simulations and its modelling ${ }^{29}$. The first LES of the expansion-compression corner flow was conducted by Knight et al. ${ }^{30}$ at $\mathrm{Mach}=2.88, \beta=25^{\circ}$ and $\mathrm{Re}_{\delta}=20000$. The flow conditions were similar to that of the experiments conducted by Zheletovodov et al..$^{21,23,24,31}$, apart from a lower Reynolds number used due to the limitation of computing resources. Knight et al. ${ }^{30}$ adopted Monotone Integrated Large Eddy Simulation (MILES) method with the second-order Godunov scheme for the spatial discretization on an unstructured tetrahedral mesh. Their MILES results have shown general good agreement with the experimental measurements. Recently El-Askary ${ }^{32}$ conducted another LES with the same flow condition as that of Knight et al. ${ }^{30}$ Again, the MILES methodology was adopted together with the second-order upwind-biased scheme. These two previous LES studies were both conducted at a low Reynolds number of $\operatorname{Re}_{\delta}=20000$ with similar numbers of mesh cells. The LES of El-Askary ${ }^{32}$ has shown better predictions of velocity profiles, mean wall pressure and wall skin friction coefficients than those of Knight et al. ${ }^{30}$, probably due to the improved mesh quality. 
In general, the SGS model used in the LES of a compressible flow containing shock-waves can be very misleading, due to the numerical dissipations arising from the shock-capturing scheme, which would make the effect of the SGS model dominated by the numerical scheme ${ }^{33}$. This was partly the reason for the two previous LES studies of Knight et al. ${ }^{30}$ and ElAskary $^{32}$, discarding the SGS model and taking the MILES methodology instead. With the development of computer power and computational technology, it is now possible to perform higher-order DNS for this flow problem on finer meshes to obtain more detailed and accurate results at low to moderate Reynolds numbers.

In this paper, we are going to present a series of DNS studies of supersonic Mach 2.9 flows over an expansioncompression corner of a $25^{\circ}$ deflection angle. Three Reynolds numbers considered are $\operatorname{Re}_{\delta}=20000,40000$ and 80000 , in which the case of $\operatorname{Re}_{\delta}=20000$ corresponds to the flow conditions of the two previous LES studies. ${ }^{30,32}$ The cases of Re $\delta=$ 40000 and 80000 are chosen to approach the flow conditions of the experiments of Zheltovodov et al. ${ }^{21-24,31}$, which have been presented in a generalized view by Knight et al. ${ }^{2,30}$. The DNS results will be validated by comparing with experimental measurements and other available numerical simulation data. The detailed turbulence structures and flow statistics will be analysed, and the Reynolds number effect will be discussed accordingly.

\section{COMPUTATIONAL SETUP}

\section{A. Governing equations}

The three-dimensional unsteady compressible Navier-Stokes (N-S) equations in a general, time-invariant, curvilinear coordinate system are solved numerically in the frame of the high-order finite difference method. The N-S equations are nondimensionalized with the reference length $L_{0}$, and velocity $u_{0}$, temperature $T_{0}$, density $\rho_{0}$, and dynamic viscosity $\mu_{0}$ of the incoming free stream flow. The resulting dimensionless parameters are Reynolds number $\operatorname{Re}=\rho_{0} u_{0} L_{0} / \mu_{0}$ and Mach number $M=u_{0} / \sqrt{\gamma R T_{0}}$. A constant Prandtl number $\operatorname{Pr}=\mu C p / k=0.72$ is used, where $C p=\gamma R /(\gamma-1)$ is the specific heat capacity of gas at a constant pressure and $k$ is the thermal conductivity. Parameters $R$ and $\gamma$ are the specific gas constant and the specific heat capacity ratio, which are set to $R=287.1 \mathrm{~J} /(\mathrm{Kg} \cdot \mathrm{K})$ and $\gamma=1.4$, respectively This set of $\mathrm{N}-\mathrm{S}$ equations can be written in a strong conservation form as

$$
\frac{\partial(J \boldsymbol{Q})}{\partial t}+\frac{\partial\left(\widehat{\boldsymbol{E}}-\widehat{\boldsymbol{E}}_{v}\right)}{\partial \xi}+\frac{\partial\left(\widehat{\boldsymbol{F}}-\widehat{\boldsymbol{E}}_{v}\right)}{\partial \eta}+\frac{\partial\left(\widehat{\boldsymbol{G}}-\widehat{\boldsymbol{G}}_{v}\right)}{\partial \zeta}=0
$$

where the coordinate transformation between the physical domain $(x, y, z)$ and the computational domain $(\xi, \eta, \zeta)$, can be described by the following equations:

$$
x=x(\xi, \eta, \zeta), y=y(\xi, \eta, \zeta), z=z(\xi, \eta, \zeta)
$$


In Eq. (1), $J=|\partial(x, y, z) / \partial(\xi, \eta, \zeta)|$ is the Jacobian of the coordinate transformation and $\boldsymbol{Q}=[\rho, \rho u, \rho v, \rho w, E]^{T}$ is the solution vector. The primary variables are the density $\rho$, the velocity component $u, v, w$, and the total energy $E$. The static temperature $T$ and the pressure $P$ are related to the density $\rho$ via the equation of state of the ideal gas law $P=\rho T /\left(\gamma M^{2}\right)$.

The convection and diffusion terms in (1) are respectively defined as

$$
\widehat{\boldsymbol{E}}=\left[\begin{array}{c}
\rho \widehat{U} \\
\rho u \widehat{U}+\hat{\xi}_{x} P \\
\rho v \widehat{U}+\hat{\xi}_{y} P \\
\rho w \widehat{U}+\hat{\xi}_{z} P \\
(E+P) \widehat{U}
\end{array}\right], \widehat{\boldsymbol{F}}=\left[\begin{array}{c}
\rho \widehat{V} \\
\rho u \widehat{V}+\hat{\eta}_{x} P \\
\rho v \widehat{V}+\hat{\eta}_{y} P \\
\rho w \widehat{V}+\hat{\eta}_{z} P \\
(E+P) \hat{V}
\end{array}\right], \widehat{\boldsymbol{G}}=\left[\begin{array}{c}
\rho \widehat{W} \\
\rho u \widehat{W}+\hat{\zeta}_{x} P \\
\rho v \widehat{W}+\hat{\zeta}_{y} P \\
\rho w \widehat{W}+\hat{\zeta}_{z} P \\
(E+P) \widehat{W}
\end{array}\right],
$$

and

$$
\widehat{\boldsymbol{E}}_{\boldsymbol{v}}=\left[\begin{array}{c}
0 \\
\hat{\xi}_{x i} \tau_{i 1} \\
\hat{\xi}_{x i} \tau_{i 2} \\
\hat{\xi}_{x i} \tau_{i 3} \\
\hat{\xi}_{x i} b_{i}
\end{array}\right], \widehat{\boldsymbol{F}}_{\boldsymbol{v}}=\left[\begin{array}{c}
0 \\
\hat{\eta}_{x i} \tau_{i 1} \\
\hat{\eta}_{x i} \tau_{i 2} \\
\hat{\eta}_{x i} \tau_{i 3} \\
\hat{\eta}_{x i} b_{i}
\end{array}\right], \widehat{\boldsymbol{G}}_{\boldsymbol{v}}=\left[\begin{array}{c}
0 \\
\hat{\zeta}_{x i} \tau_{i 1} \\
\hat{\zeta}_{x i} \tau_{i 2} \\
\hat{\zeta}_{x i} \tau_{i 3} \\
\hat{\zeta}_{x i} b_{i}
\end{array}\right] .
$$

Here, $\hat{\xi}_{x}=J \partial \xi / \partial x$. The standard Einstein summation notation is used and notations $x_{i}, u_{i}$ and $\xi_{i}, i=1,2,3$ are adopted to represent $(x, y, z),(u, v, w)$ and $(\xi, \eta, \zeta)$, respectively, and the contravariant velocity components and the total energy are written as

$$
\widehat{U}=\hat{\xi}_{x i} u_{i}, \hat{V}=\hat{\eta}_{x i} u_{i}, \widehat{W}=\hat{\zeta}_{x i} u_{i}
$$

and

$$
E=\frac{1}{2}\left(\rho u_{i} u_{i}\right)+\frac{\rho T}{\gamma(\gamma-1) M^{2}} .
$$

The stress tensor and the heat flux vector are expressed as

$$
\tau_{i j}=\frac{\mu}{\operatorname{Re}}\left(\frac{\partial u_{i}}{\partial \xi_{k}} \frac{\partial \xi_{k}}{\partial x_{j}}+\frac{\partial u_{j}}{\partial \xi_{k}} \frac{\partial \xi_{k}}{\partial x_{i}}-\frac{2}{3} \delta_{i j} \frac{\partial u_{l}}{\partial \xi_{k}} \frac{\partial \xi_{k}}{\partial x_{l}}\right)
$$

and

$$
b_{i}=u_{j} \tau_{i j}+\frac{\mu}{\operatorname{PrRe}(\gamma-1) M^{2}} \frac{\partial T}{\partial \xi_{k}} \frac{\partial \xi_{k}}{\partial x_{i}} .
$$

The dynamic viscosity coefficient $\mu$ is calculated via the Sutherland law:

$$
\mu=T^{1.5} \frac{T_{S} / T_{0}+1}{T+T_{S} / T_{0}}
$$

where $T_{0}=104.4 \mathrm{~K}$ and $T_{S}=110.4 \mathrm{~K}$ according to the experiment condition $^{31}$.

\section{B. Numerical scheme}

To properly resolve small-scale turbulence structures in the compressible turbulence with shock-waves, the convection terms of the N-S equations are solved by using the newly developed seventh-order low-dissipative monotonicity-preserving 
(MP7-LD) scheme ${ }^{34}$, which is optimized from the original MP scheme of Suresh and Huynh ${ }^{35}$ by reducing both the linear dissipation and the nonlinear error. This scheme has been successfully used in DNS of shock-wave/isotropic turbulence interaction $^{34}$ and impinging shock-wave/flat-plate boundary layer interaction ${ }^{36}$ and it was proved that the MP7-LD scheme has the same ability as the original MP scheme in capturing shock waves and with a better performance in resolving smallscale turbulent fluctuations ${ }^{34,36}$.

The diffusion terms of N-S equations are solved with the sixth-order compact scheme ${ }^{37}$. The primitive velocity components $u_{i}$ and temperature variable $T$ are differentiated firstly to formulate the stress tensor and the heat flux vector at each node point. The diffusion terms are then computed with another application of the sixth-order compact scheme ${ }^{38}$. This method is more efficient than direct calculation of the second-order derivatives ${ }^{39}$, although the latter method can be numerically more stable. After all the spatial terms are solved, the third-order TVD Runge-Kutta method ${ }^{40}$ is used for the time integration. The geometry matrix of the grid transformation is also calculated with the sixth-order compact central scheme in the conservation form ${ }^{41}$ to preserve the accuracy of the solution.

\section{Boundary condition}

In DNS/LES of turbulent boundary layers, the generation of the inlet turbulence fluctuations is an important issue and some efforts have been made in the past. In the present research, the digital filter method proposed by Touber and Sandham ${ }^{42,43}$ is used to generate synthetic inflow turbulence. This method is very competitive compared with the widely used rescale-reintroduce method ${ }^{44,45}$ in terms of the domain requirement for the transition region ${ }^{46}$ and thus has the benefit of avoiding any further constraints such as numerical periodicity.

After the random inflow fluctuations are generated by using the digital filter method with prescribed Reynolds stress components fitted from the incompressible DNS data of $\mathrm{Wu}$ and Moin ${ }^{47}$, they are super-imposed onto mean velocity and temperature profiles of the turbulent boundary layer and the supersonic inflow condition is then used to prescribe the flow variables at the inlet plane, except for the subsonic portion of the boundary layer, where the pressure is extrapolated from the inner grid points.

At the far-field and the outlet plane, the generalized non-reflecting boundary condition is used to calculate flow variables $^{48,49}$. Along the bottom wall, the isothermal non-slip condition with the fixed wall temperature $T_{W}=2.51 T_{0}$ is used at the wall. The periodic condition is used in the spanwise direction.

\section{Computational domain and flow conditions}

The computational domain is sketched in FIG. 2, normalized with the nominal boundary layer thickness $\delta_{r e f}$ at the reference plane $x=0$ (not the inlet plane) and the expansion corner is placed $4 \delta_{\text {ref }}$ downstream of the reference plane. In 
order to be consistent with the experiments of Zheltovodov et al. ${ }^{21,24,31}$ as well as the LES of Knight et al. ${ }^{30}$ and El-Askary ${ }^{32}$, the deflection angle of the backward-facing ramp is set to be $\beta=25^{\circ}$, and the vertical distance between the two horizontal flat plates is $h=3 \delta_{\text {ref }}$. The detailed information about the domain sizes and meshes of three case studies is given in TABLE I. Unless otherwise stated, $\delta_{\text {ref }}$ is used as the reference length scale hereafter. Case 1 has a slightly finer mesh resolution than that of the Case 2 and Case 3, and they all satisfy the DNS spatial resolution requirement recommended by Sagaut ${ }^{50}$ for the wall-bounded turbulent flows.

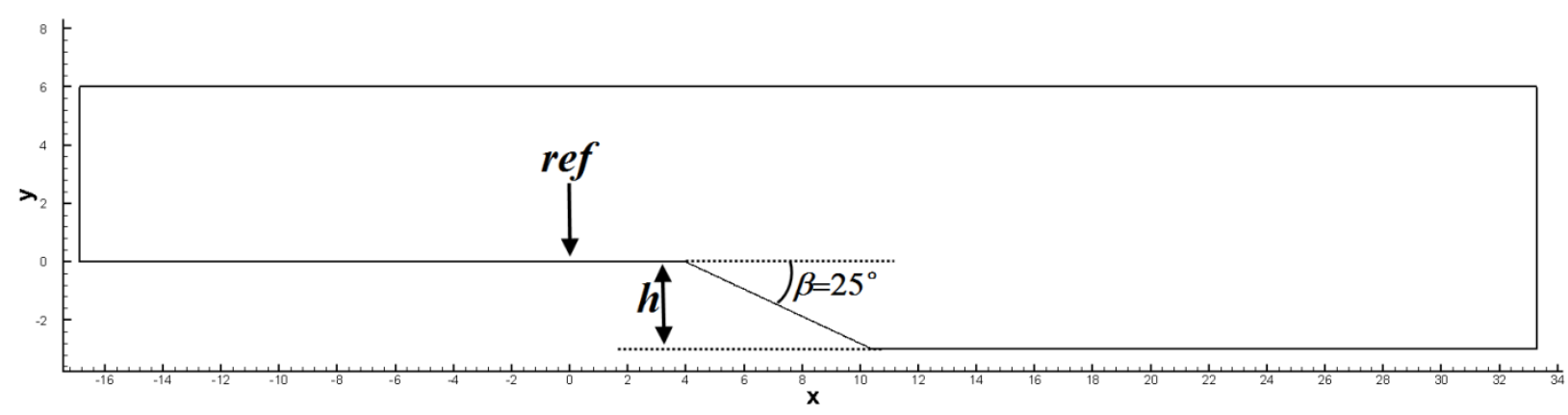

FIG. 2. The sketch of the Computation domain of Case 1.

TABLE I. Domain size and mesh parameters.

\begin{tabular}{cccccccccc}
\hline \hline & $R e_{\delta_{\text {ref }}}$ & $x_{0}$ & $x_{\max }$ & $y_{\max }$ & $Z \max$ & $N_{x} \times N_{y} \times N_{z}$ & $\left(\Delta x_{\max }^{+}, \Delta x_{\min }^{+}\right)$ & $\left(\Delta y_{1}^{+}, \Delta y_{e}^{+}\right)$ & $\Delta z^{+}$ \\
\hline Case 1 & 19983.8 & -16.9 & 33.2 & 6 & 6 & $1420 \times 120 \times 256$ & $(6.7,1.3)$ & $(0.68,7.3)$ & 4.3 \\
Case 2 & 40934.2 & -13.6 & 33.4 & 6 & 5 & $2020 \times 120 \times 300$ & $(8.1,1.6)$ & $(0.52,15.8)$ & 5.7 \\
Case 3 & 80051.0 & -11.9 & 23.7 & 6 & 3 & $2620 \times 200 \times 400$ & $(9.3,1.9)$ & $(0.72,17.2)$ & 7.5
\end{tabular}

* $x_{0}$ denotes the location of the inlet plane. $x_{\max }, y_{\max }, z_{\max }$ stand for the locations of the outlet plane, far-field plane and spanwise size. $N_{x} \times N_{y} \times N_{z}$ indicates the number of mesh points in each direction. $\Delta x_{\max }^{+}$and $\Delta x_{\min }^{+}$stand for the maximum and minimum mesh spaces in the $x$ direction. $\Delta y_{1}^{+}$and $\Delta y_{e}^{+}$means the mesh space in the $y$ direction at the first point away from wall and the edge of the boundary layer for the reference location.

By referencing the numerical experiments of Touber and Sandham ${ }^{42}$ and Morgan et al. ${ }^{46}$, the distance between the inlet plane and the reference plane $x_{0}$ is sufficient long for the synthetic fluctuations being evolved to be a fully-developed turbulence in the present studies. The spanwise domain is also wide enough to contain several large-scale flow structures such as the Görtler vortices ${ }^{21,51}$. To eliminate the possible reflection of numerical disturbances from the boundaries, two sponge layers each with 15 and 20 layers of stretched meshes and a low-order Laplacian filter proposed by Gloerfelt and Lafon $^{52}$ are incorporated near the far field and the outlet planes, respectively. 
The present DNS mesh (as shown in FIG. 3) has straight and perpendicular lines in all three directions, except for corner regions, where the mesh lines are gradually curved towards the normal of the ramp surface in order to achieve the balance between the grid orthogonality and the grid smoothness. The mesh is uniformly distributed in the spanwise ( $z$ ) direction and hyperbolically stretched in the wall-normal direction to increase the resolution in the near wall region with the formula: $y(\eta)=\mathrm{y}_{\max }\left[1+a \cdot \tanh \left(b \cdot \frac{\eta-\eta_{\max }}{\eta_{\max }}\right)\right], \eta=0,1,2, \ldots, \eta_{\max }, b=\operatorname{arctanh}(1 / a)$, with the parameter $a$ controlling the first point away from the wall. In the $\mathrm{x}$ direction, the mesh is smoothly adjusted to enhance the resolution in the SWTBLI region, where small-scale turbulence could be existent. The parameters of the adopted meshes are listed in TABLE I.

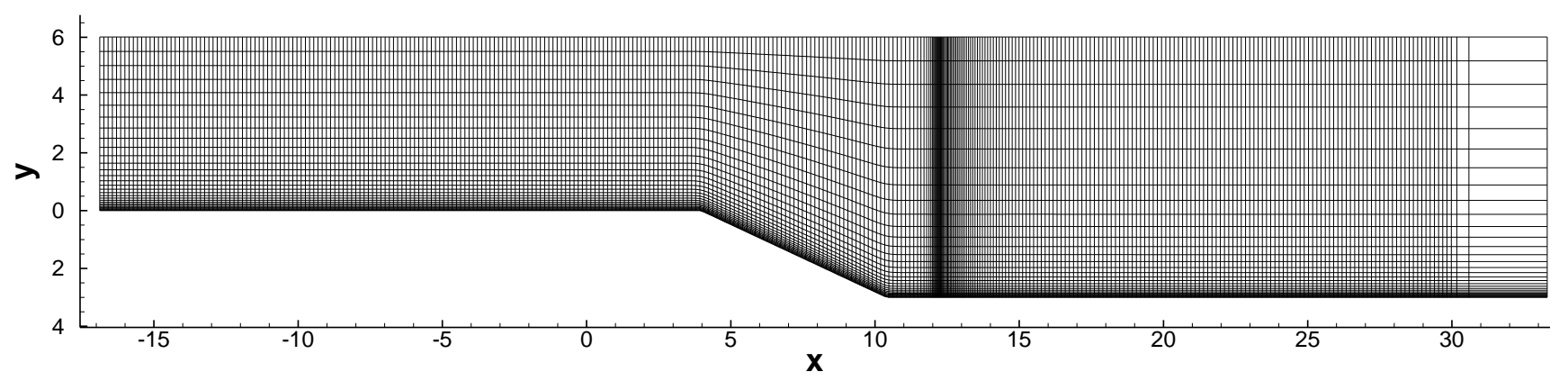

FIG. 3. A sketch of the Case 1 mesh. The mesh is plotted every 4 points in both $x$ and $y$ directions for the convenience of visualization.

The non-dimensional time steps $\frac{\Delta t}{\delta_{r e f} / u_{0}}$ for Case 1, Case 2 and Case 3 are 0.0032, 0.0019 and 0.0014 respectively. The statistics are acquired by averaging the flow field every 50 time steps after the flow reaches statistically converged results and a total of 12550, 26200 and 13400 instantaneous flow field samples are collected for Case 1, Case 2 and Case 3, respectively. The flow is expected to be fully developed at the reference plane $x=0$, at which the boundary layer parameters are listed in TABLE II.

TABLE II. Summary of boundary layer parameters at the reference plane.

\begin{tabular}{|c|c|c|c|c|c|c|c|c|}
\hline & $M$ & $\delta$ & $\delta^{*}$ & $\theta$ & $R e_{\delta}$ & $R e_{\delta^{*}}$ & $R e_{\theta}$ & $C f$ \\
\hline Case 1 & 2.9 & 1.0 & 0.45 & 0.077 & 19983.8 & 8950.8 & 1531.8 & 0.00224 \\
\hline Case 2 & 2.9 & 1.0 & 0.42 & 0.076 & 40934.2 & 17203.8 & 3090.4 & 0.00186 \\
\hline Case 3 & 2.9 & 1.0 & 0.43 & 0.076 & 80051.0 & 34567.2 & 6140.7 & 0.00138 \\
\hline
\end{tabular}

The time histories of instantaneous pressure $P$ and the evolutions of the first to third-order statistics i.e. $\bar{P}, \overline{p^{\prime 2}}$ and $\overline{p^{\prime 3}}$ of the two representative points (point A locates in the log-layer of the upstream undisturbed boundary layer and point B locates in the centre of the separation bubble) of Case 1 are plotted in FIG. 4. For Case 1, the simulation reaches the statistically stationary state at about $t=230$, after which statistics are collected every 50 steps. The evolution of statistics is similar with those in the LES of the corner separation by Gao et al. ${ }^{53}$. For both points, the mean and second-order statistics have converged to steady states before the end of the simulation at $t=2740$, although the third-order statistic still presents a light 
oscillation at the end of the simulation. The convergence properties of Case 2 and Case 3 are similar with that of Case 1; therefore the steady states have been reached at least for the first and second-order statistics.

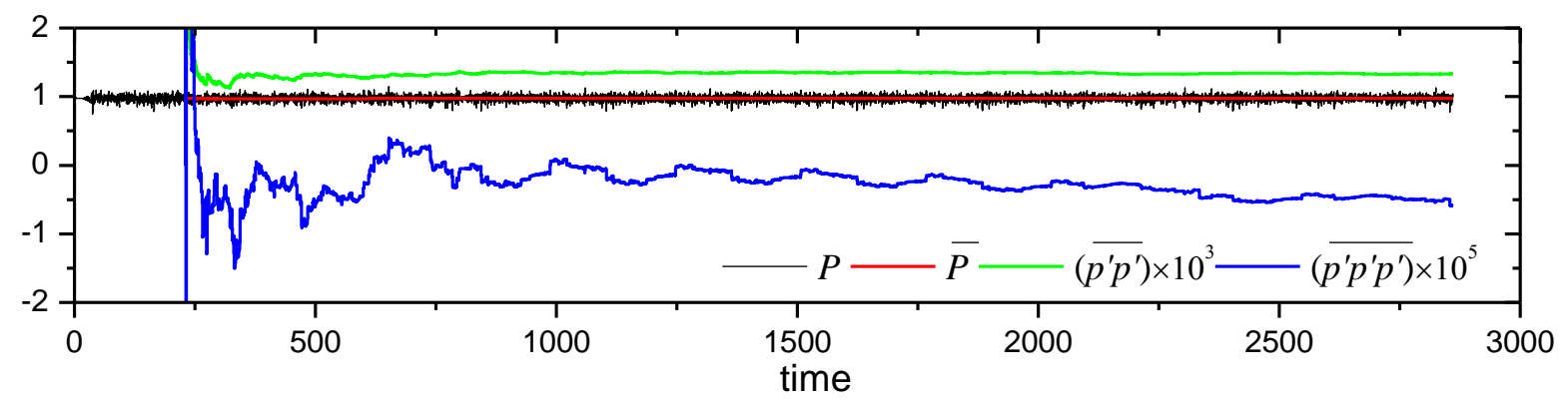

(a)

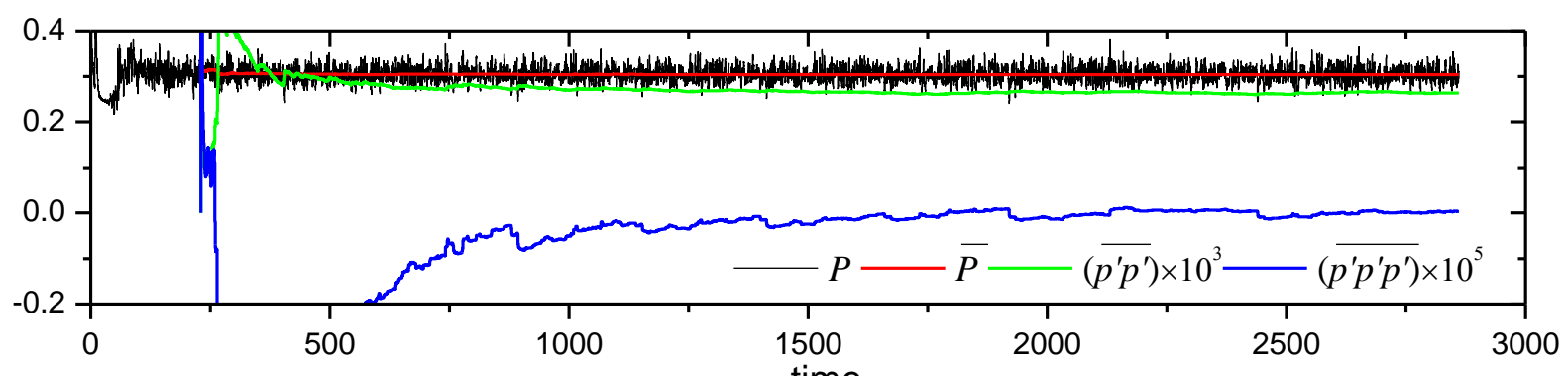

(b)

FIG. 4. Statistical convergence at two representative points A and B. (a): Point A; (b): Point B.

\section{RESULTS AND DISCUSSIONS}

\section{A. Validation and general properties of the flow field}

To validate the present DNS results, we firstly compared the velocity profile at $x=2$ in FIG. 5 (a), where the boundary layer is in the equilibrium state with the available experimental measurements of Zheltovodov et al. ${ }^{31}$ at the same Mach number and the Reynolds number of $R e_{\delta}=80000$. It shows that an overall good agreement between DNS results and the measurement has been achieved. The van Driest transformed non-dimensional mean velocity in wall unit $u_{v d}^{+}$is also plotted in FIG. 5 (b), together with the law of wall and the measurements of incompressible boundary layers of Murlis et al. ${ }^{54}$ and Erm and Joubert ${ }^{55}$. The mean flow variables are calculated via Favré averaging, defined as $\langle f\rangle=\overline{\rho f} / \bar{\rho}$, in which $f$ is a general variable and $\bar{f}$ stands for the mean value. The fluctuations are then defined as $f^{\prime \prime}=f-\langle f\rangle$ and $f^{\prime}=f-\bar{f}$. It can be seen from FIG. 5 (b) that the van Driest transformed velocity profile in the linear sub-layer and the log-layer perfectly matches the incompressible law of the wall with the standard von Kármán constant. With the increase of the Reynolds number, the log layer extends and the wake-layer becomes higher. The overall agreement between the present DNS and the incompressible flow measurements is satisfactory in the sub-layer and the log-layer, and the discrepancy in the wake-layer could be attributed to the Reynolds number effects. In FIG. 5 (c), the velocity profile in the non-equilibrium recovery zone of 
Case 3 is compared with the measurement data of Zheltovodov et al. ${ }^{31}$, in which the flow conditions are the same with the present case. Again, the present DNS prediction and the experimental measurement match well with each other.

(a)

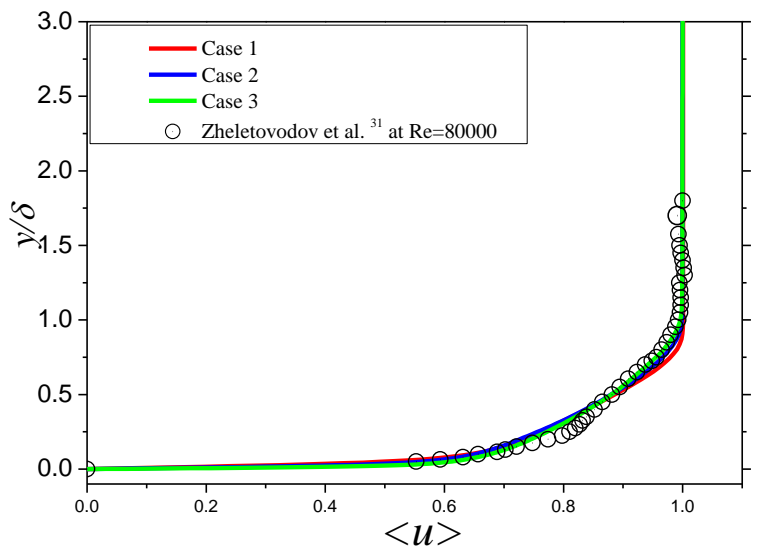

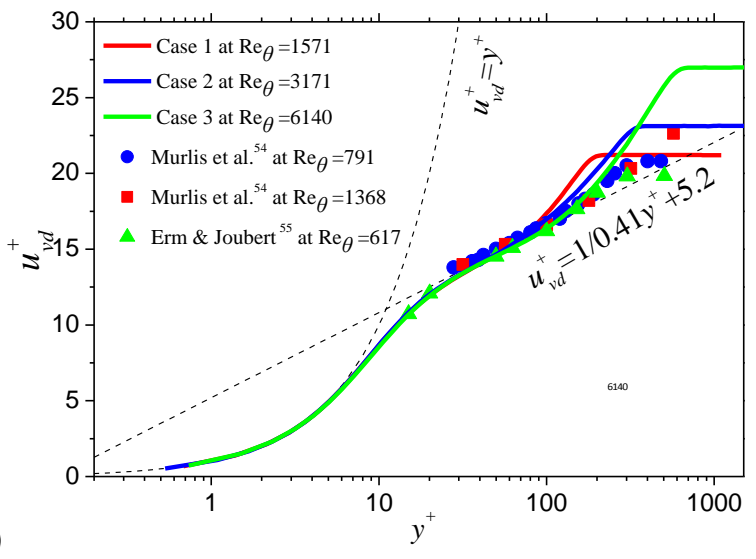

(b)

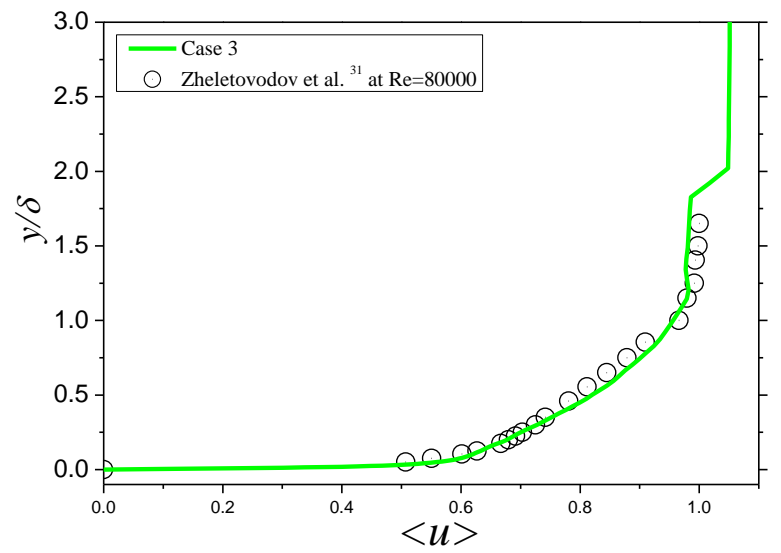

(c)

FIG. 5. Mean velocity profile at $x=2$ (a, b) and $x=19$ (c). (a) and (c) are plotted in the outer scaling and (b) is plotted in inner scaling. The experimental data in (a) and (c) are measured at $9 \mathrm{~mm}$ and $30 \mathrm{~mm}$ upstream and downstream of EC respectively with the reference length of $\delta_{\text {ref }}=2 \mathrm{~mm}$.

The density scaled non-dimensional root mean square (RMS) of velocity fluctuation intensities $\frac{1}{u_{\tau}} \sqrt{\frac{\bar{\rho}}{\overline{\rho_{w}}}}\left\langle u_{i}^{\prime \prime} u_{i}^{\prime \prime}\right\rangle$ at $x=2$ are plotted in FIG. 6, together with the published data of the incompressible experimental measurements of Purtell et al. ${ }^{56}$ and Erm and Joubert ${ }^{55}$, the incompressible DNS of Spalart ${ }^{57}$ and Wu and Moin ${ }^{47}$, and the compressible DNS of Mach=1.3 turbulent boundary layer of Pirozzoli et al. ${ }^{58}$.

It can be seen that the present DNS results are in good agreement with experimental measurements and other DNS predictions in both the near wall region (see FIG. 6 (a) and the outer part of the boundary layer (see FIG. 6 (b) ), when the inner and the outer scales are used respectively. 

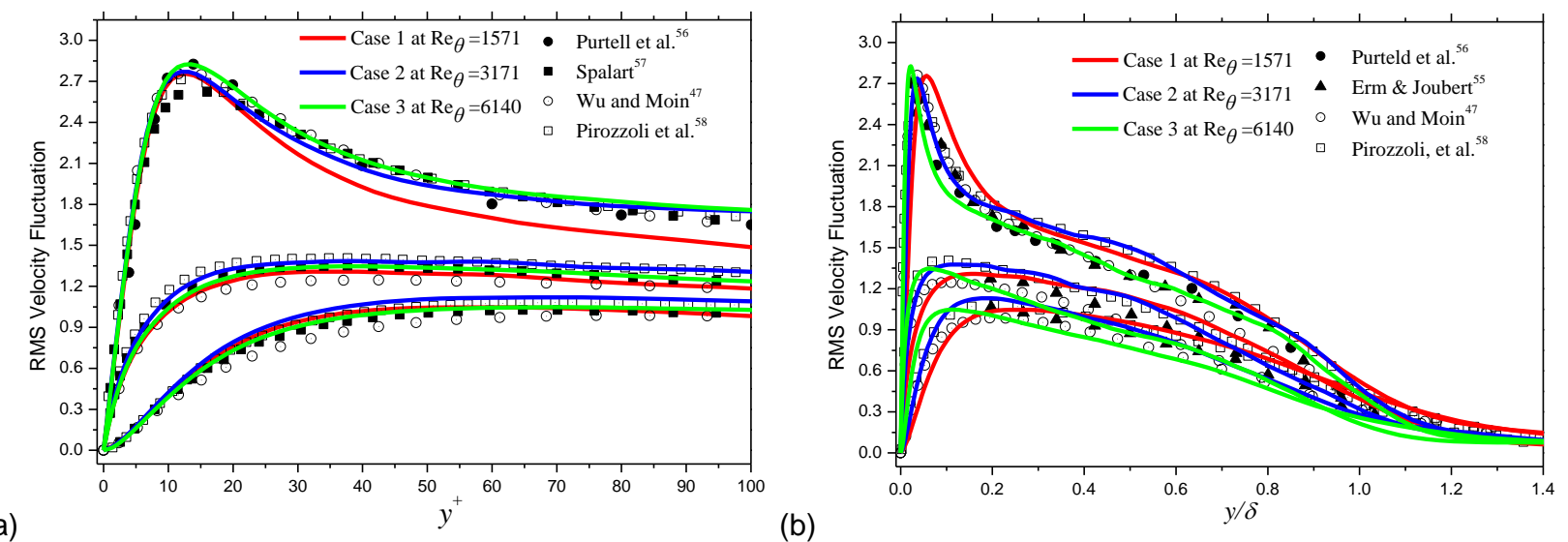

(b)

FIG. 6. RMS velocity fluctuation intensities at $x=2$ in inner scaling (a) and outer scaling (b).

The mean pressure field super-imposed with streamlines is shown in FIG. 7. It can be seen that an expansion fan is formed from the expansion corner and a $\lambda$-shock-wave system is formed around the compression corner downstream. The front leg of the $\lambda$-shock-wave system impinges at about the middle of the ramp surface and the rear leg impinges close to the compression corner. In the near-wall region, the shock-wave has been smeared and looks as a series of compression-waves. The APG generated by the strong shock-wave causes flow separation that is occurred around the compression corner and the comparison of the three cases in FIG. 7 indicates the shrinkage of the separation bubble with increasing Reynolds number.

(a)

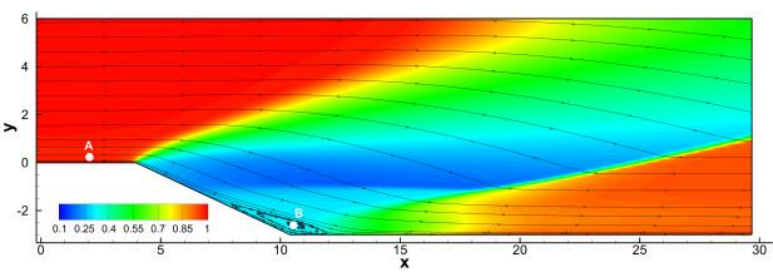

(b)

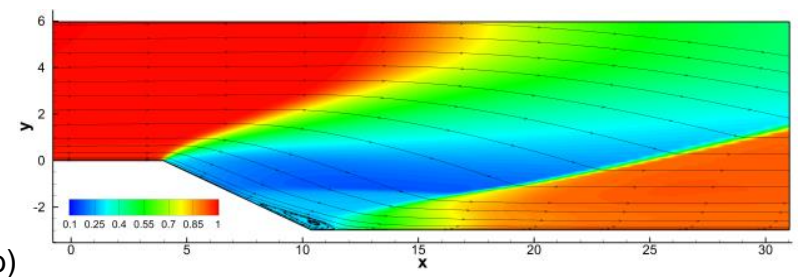

(c)

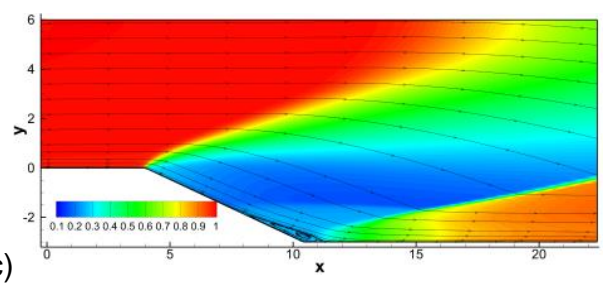

FIG. 7. The mean pressure field and streamlines of Case 1 (a), Case 2 (b) and Case 3 (c). The spots A and B in (a) mark the two monitored points.

The comparison of the mean wall pressure distributions from three present DNS as well as previous LES results ${ }^{30,32}$ and the experimental data ${ }^{31}$ generalized by Knight et al. ${ }^{30}$ are shown in FIG. 8 (a). It can be seen that, the pressure is reduced sharply at EC position due to the expansion, then reduced slowly towards the middle of the ramp, i.e. at the foot of the front shock-wave, where the wall pressure begins to rise. A pressure-plateau can be identified in the region of $8<x<11$ corresponding to the location of the separation bubble. After interaction with the rear shock-wave, the pressure is largely increased. With the increase of the Reynolds number, the level of the pressure-plateau is slightly reduced and the pressure 
after the rear shock-wave increases more rapidly, which agrees with the trend of the experimental data. Overall, the wall pressure distributions of the present cases agree very well with the experiments data and previous LES results.

From the mean wall pressure gradient $d P_{W} / d x$ distributions shown in FIG. 8 (b), we can see the two positive peaks for each case, which correspond to the locations of the front and the rear legs of the $\lambda$-shock-wave respectively. With the increase of the Reynolds number, the first peak moves slightly downstream and the second peak moves slightly upstream indicating the decrease of the angle between these two legs of the $\lambda$-shock-wave system due to the shrink of the separation bubble. The magnitude of the second peak also increases with the increase of the Reynolds number, due to the reduction of the viscous effect. The mean wall pressure gradient profiles present some oscillation after CC, especially for Case 3 , because the convergence of pressure statistics has a strict requirement on the number of samples.

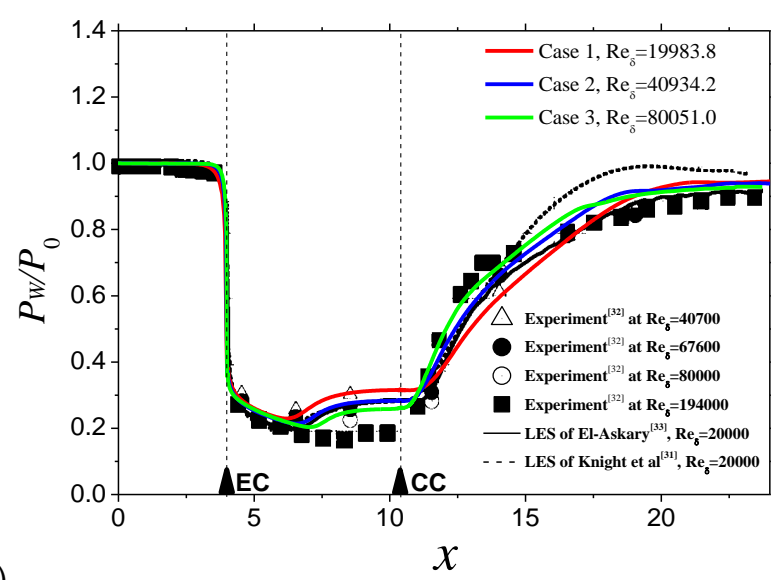

(a)

FIG. 8. Mean wall pressure distribution (a) and wall pressure gradient distribution (b). The pressure gradient is normalized with $P_{0} / \delta_{\text {ref }}$.

The wall pressure fluctuation intensity $\overline{p^{\prime} p^{\prime}}$ distributions are present in FIG. 9. It can be seen that, $\overline{p^{\prime} p^{\prime}}$ is firstly suppressed sharply while passing through EC and then the decrease of $\overline{p^{\prime} p^{\prime}}$ slows down. After interacting with the front leg of the $\lambda$-shock-wave, the wall pressure fluctuation begins to rise. Downstream of $\mathrm{CC}$, the dramatic amplification of $\overline{p^{\prime} p^{\prime}}$ can be observed and the peak of $\overline{p^{\prime} p^{\prime}}$ can be found downstream of CC. Along the ramp wall, the profiles of $\overline{p^{\prime} p^{\prime}}$ of the three cases collapse well, after CC the Reynolds number effect is clearly presented. With the increase of the Reynolds number, the peak of $\overline{p^{\prime} p^{\prime}}$ reaches a greater value and moves closer to the corner, which is similar with the trend of the wall pressure gradient seen in FIG. 8 (b). This indicates the strong influence of SWTBLI on the wall pressure fluctuation strength, and with the increase of the Reynolds number, the shock-wave will penetrate deeper into the boundary layer and therefore induces larger wall pressure gradient and wall pressure fluctuations. A slight oscillation of $\overline{p^{\prime} p^{\prime}}$ upstream EC can be observed due to the difficulty in converging high-order pressure statistics. 


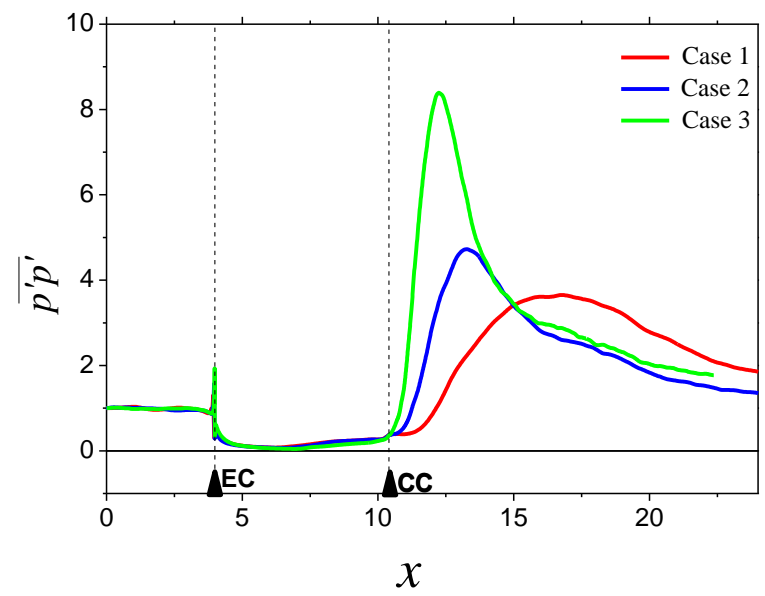

FIG. 9. Wall pressure fluctuation intensity $\overline{p^{\prime} p^{\prime}}$ normalized with its value at the reference plane.

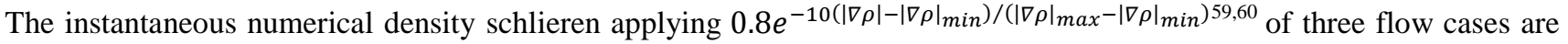
shown in FIG. 10. The experimental schlieren of Zheltovodov et al. ${ }^{21}$ at $R e_{\delta}=190000$ is also given for qualitative comparison. The $\lambda$-shock-wave system can be seen clearly from the DNS schlieren. It can be seen that the DNS and the experiment produce similar flow patterns, and also we can observe the weakening of the turbulence fluctuations with the increase of their coherent structures during the expansion process.

(a)
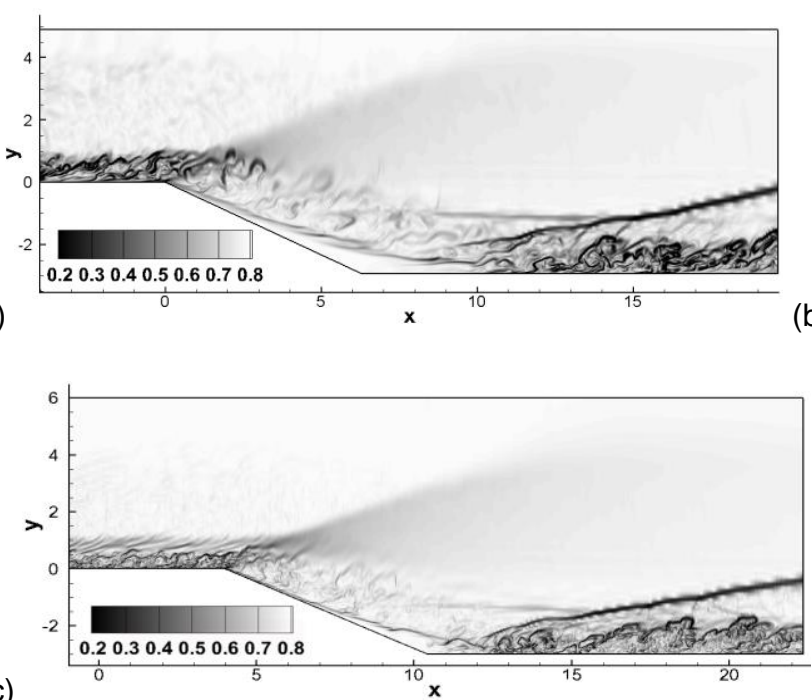

(b)

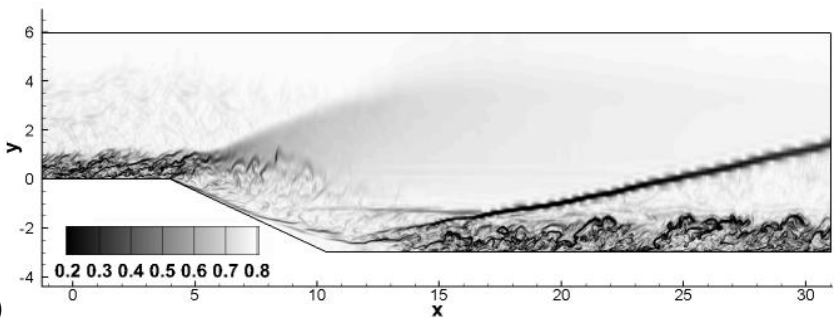

(d)

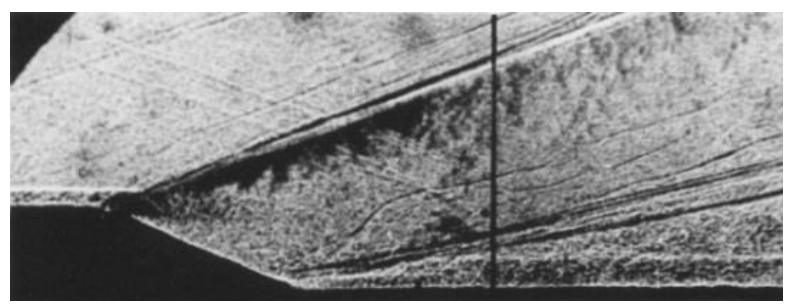

FIG. 10. The instantaneous density schlieren of (a) Case 1, (b) Case 2, (c) Case3 and (d) the experimental observation of Zheltovodov et al. ${ }^{14}$ at $\operatorname{Re}_{\delta}=190000$.

The mean skin friction distributions $C f$ are plotted in FIG. 11, in which $C f$ is calculated via the formula:

$$
C f=\frac{\left\langle\tau_{i j}\right\rangle \mid W_{W_{j} m_{i}}}{\frac{1}{2} \rho_{0} u_{0}^{2}}
$$


where $\left.\left\langle\tau_{i j}\right\rangle\right|_{W}$ is the mean viscous stress tensor at the wall surface, $n_{j}$ and $m_{i}$ are the vector units normal and parallel to the wall surface respectively.

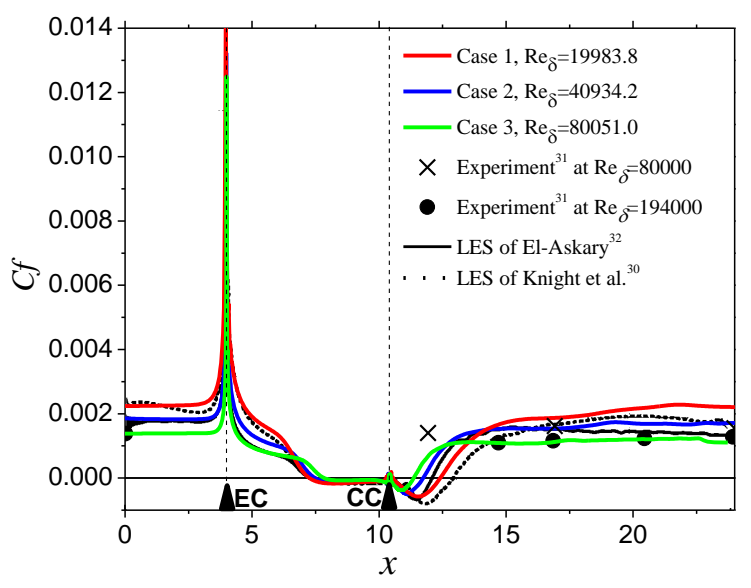

FIG. 11. Comparison of mean skin friction distributions.

The skin friction line has shown a strong spike at EC location, which is caused by the sudden change of the surface geometry. After the spike, the skin friction gradually decreases and at about the middle of the ramp, the skin friction exhibits a clear plateau region with negative values, which indicates the existence of the separation region with reverse flows. The level of plateau predicted by present DNS agrees very well with other published data at different Reynolds numbers, indicating insensitivity of flow Reynolds number in this region. The separation point also agrees with the first positive peak of the wall pressure gradient around $x=7.5$, see FIG. 8 (b), which is the location of the foot of the front shock-wave. With the increase of the Reynolds number, the separation point indicated with $C f=0$, moves slightly towards $C C$ location. Downstream of $\mathrm{CC}$, the skin friction further decreases with the increase of the adverse pressure gradient and reaches the minimum value as often observed in most shock-wave induced flow separation scenarios. After that, $C f$ begins to increase and the reattachment point can be also identified by $C f=0$. Further downstream, the increase of $C f$ slows down, which indicates the recovery of the turbulent boundary layer towards another equilibrium state.

Based on the skin friction distributions, it can be confirmed that, with the increase of Reynolds number, there is reduction of the flow separation size, forward movement of the reattachment point, and faster recovery of the skin friction downstream of the reattachment. In general, the predicted skin frictions of present DNS are in good agreements with the experimental data of Zheltovodov et al. ${ }^{21}$ as well as previous LES results ${ }^{30,32}$. Comparing all the simulation results, it can be seen that the discrepancies are mainly presented in the region downstream of the compression corner location, where the flow is in the strong non-equilibrium state due to SWTBLI. The difference of the separation bubble size and the reattachment point could 
be primarily due to the Reynolds number effect. As there is limited experimental data available in this region, it is not possible to assess which simulation produces better prediction of the skin friction.

The Stanton number $S t$ is plotted in FIG. 12, in which $S t$ is defined as

$$
S t=\left.\mu_{W} \frac{\partial\langle T\rangle}{\partial y}\right|_{W} \frac{1}{\rho_{0} u_{0}\left(T_{t}-T_{W}\right) \operatorname{PrRe}}
$$

where $T_{t}=T_{0}\left(1+\frac{1}{2}(\gamma-1) M^{2}\right)$ is the total temperature. The Stanton number is reduced to negative values during the expansion process and then increased to positive values at the foot of the front shock-wave. The peak of the Stanton number can be found immediately downstream of the reattachment point, which indicates the turbulence generated in SWTBLI may have great contributions to the wall heat flux. Upstream of the compression corner, a near plateau region can be identified and its value seems also independent of the Reynolds number. For the higher Re case, the increase of $S t$ after the CC location is faster, but for lower Re case it produces larger values of $S t$ for the peak and its downstream region, which is consistent with the evolution of the skin friction coefficient. Further downstream, the Stanton number also recovers towards the equilibrium state and with the higher Reynolds number the recovery process is faster. Comparing to that of the skin friction, the recovery of Stanton number takes a longer distance, which means the re-establishment of the thermal equilibrium state may happen further downstream after the flow reattachment.

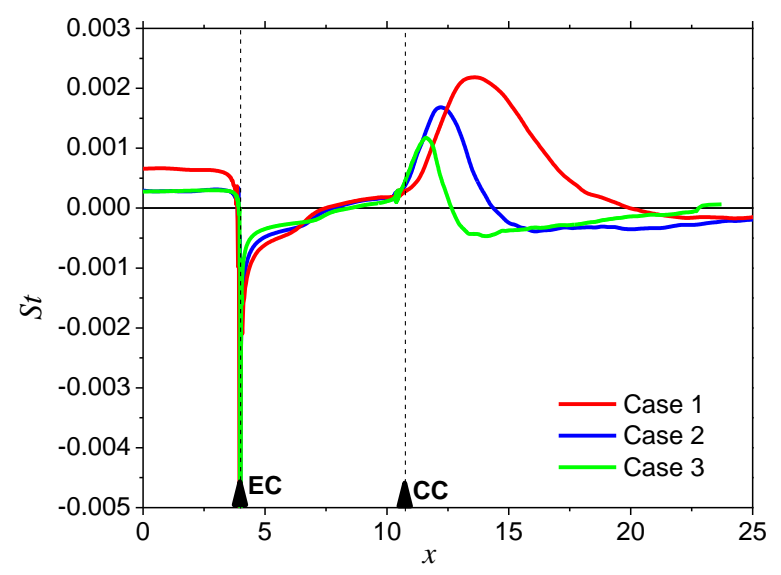

FIG. 12. The distributions of the Stanton number.

\section{B. Separation property}

As reported by Zheltovodov et al. ${ }^{21}$ as well as Roshko and Thomke ${ }^{61}$, the appearance of the so-called Görtler vortices is an important characteristic of the expansion-compression corner flow. Görtler vortices and its generation mechanism were discovered in the laminar boundary layer by Görtler ${ }^{62}$ in 1955 and observed in conditions of its reattachment in pioneering experimental investigations by Ginoux. ${ }^{63}$ Görtler vortices arise from the instability of a boundary layer with sufficiently concave streamwise curvature, where the interaction between centrifugal force, pressure gradient, and viscosity destabilizes 
the boundary layer and the streamwise vortices, termed "Görtler vortices" will then arise as a consequence. ${ }^{64}$ Although Görtler vortices were first found in the laminar flow, the same mechanism also excites in turbulent flows. ${ }^{65,66}$ In SWTBLI flows with concave streamwise curvatures, such as compression flows, the large-scale three-dimensional structures were reported in previous experimental observations $s^{21,51,63,67-69}$ and numerical simulations s1,59,70. $^{\text {. }}$

To gain further insight into the generation of Görtler vortices, three-dimensional flow separation property is investigated. The time averaged skin friction coefficient contours and surface streamlines for Case 2 are plotted in FIG. 13. Firstly, the mean skin friction presents a two-dimensional characteristic with straight parallel wall limiting streamlines upstream of the separation region. However downstream of CC the three-dimensional characteristics can be observed, especially in the region near the reattachment line, where a total of five pairs of node and saddle-point combinations can be identified. Downstream of the reattachment line, five convergence lines can be seen. Similar flow topology which is considered as the appearance of Görtler type vortices is also observed in two-dimensional compression corner flow experiments by using surface visualization technology ${ }^{21}$ and numerical simulations by using $\mathrm{DNS}^{59}$ and $\mathrm{LES}^{51}$.

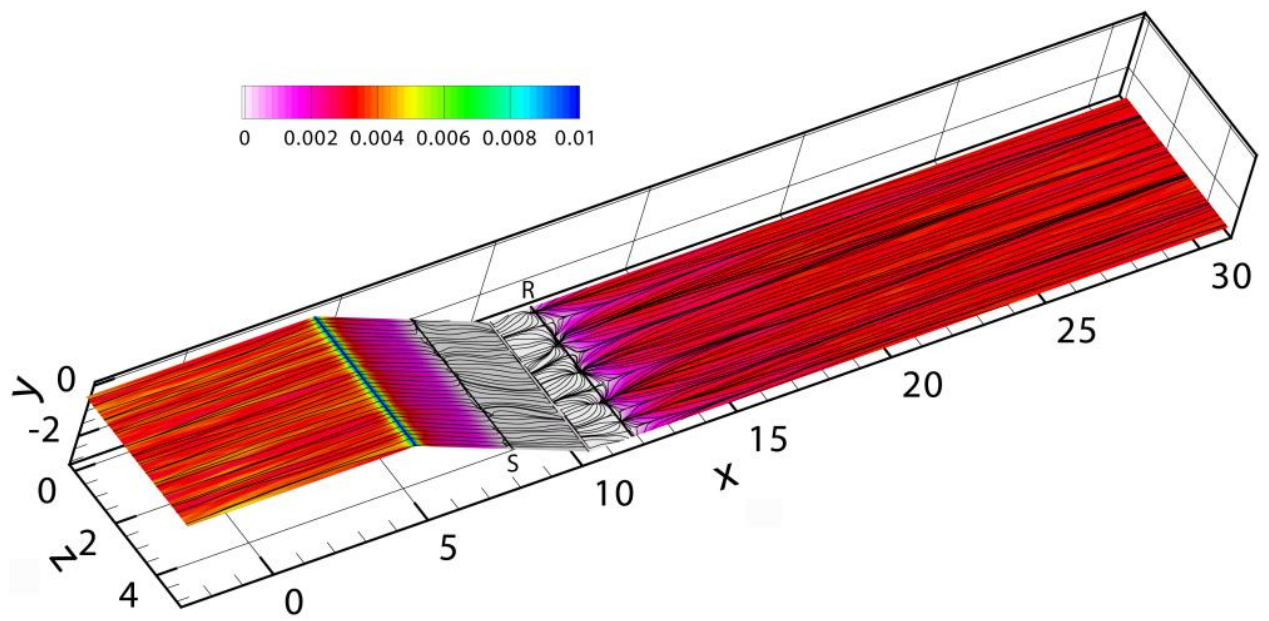

FIG. 13. Time averaged skin friction coefficient and wall limiting streamlines for Case 2.

The predicted mean surface streamlines in the 3-D region of the three cases are demonstrated in FIG. $14(\mathrm{a}-\mathrm{c})$. Downstream of the separation line, several periodic longitudinal convergence and divergence lines located along the plate width can be identified. Such periodic lines have been also observed in experiments. ${ }^{21,31}$

The topologies of the surface streamlines of the predicted three cases are almost identical (see FIG. 14(a-c)) and the spanwise distances $B$ between the neighboring convergence lines are all at a scale of $\delta_{\text {ref }}$, which are independent of the Reynolds number and smaller than that in the compression corner flow LES of Loginov et al. ${ }^{51}$, as well as other researchers (see the review of Edwards ${ }^{70}$ ) who reported a scale of $B \approx 2 \delta_{\text {ref }}$. The discrepancy between the scales of the large-scale vortex structures along the spanwise width can be attributed to the different properties of the boundary layer in the vicinity of 
the separation line in such different conditions. For the present expansion-compression corner case, the boundary layer has been strongly disturbed by the expansion fan upstream the separation bubble.
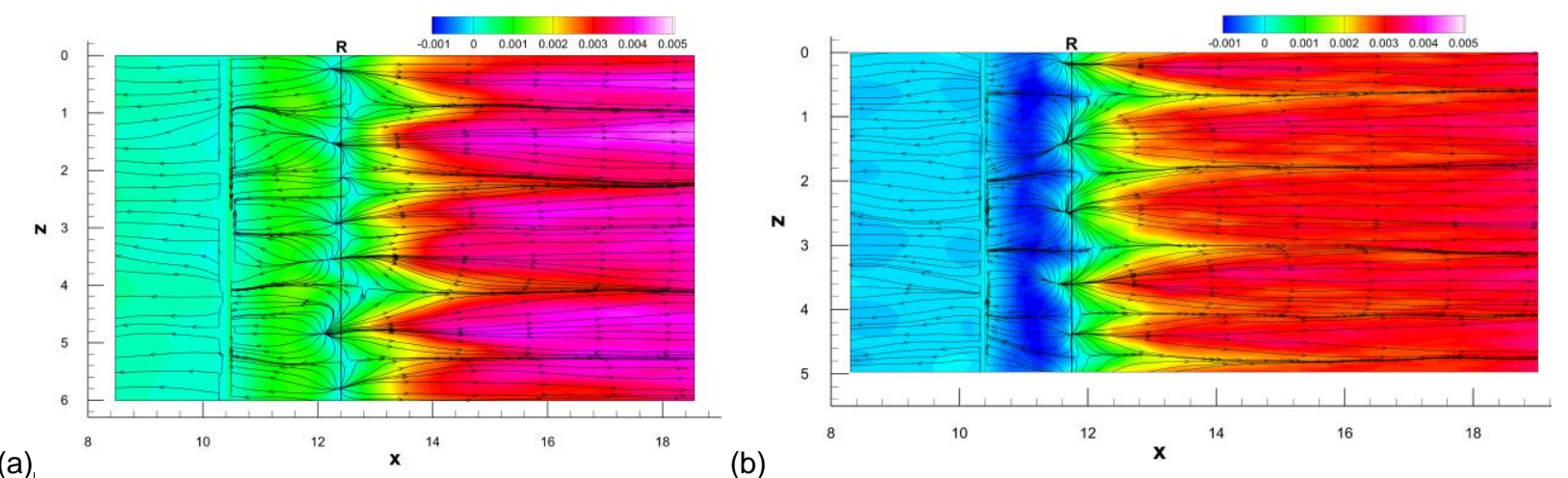

(b)

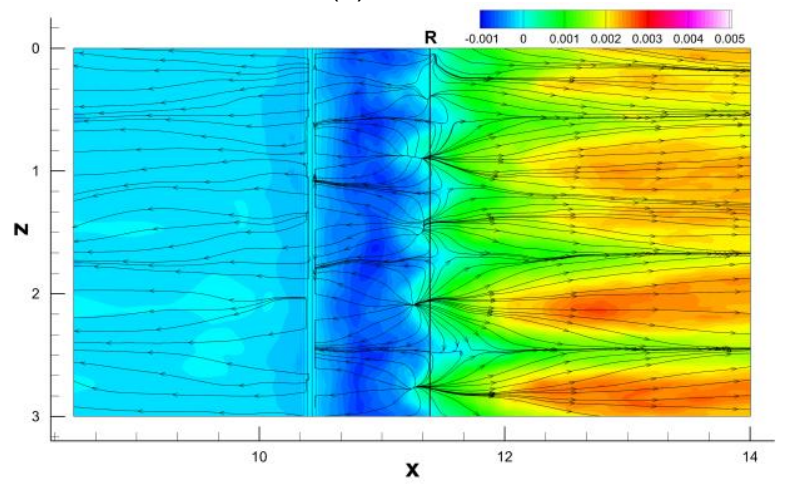

FIG. 14. Time averaged skin friction coefficient and wall limiting streamlines around the reattachment line for (a) Case 1, (b) Case 2, and (c) Case 3.

The time averaged Stanton number of Case 2 is shown in FIG. 15, in which we can see the elevated mean Stanton number around the reattachment line. Also, the distance between neighbouring high $S t$ regions is about $\delta_{r e f}$, which indicates the same mechanism of the influence of the large-scale Görtler vortices on the wall heat flux distribution as well as on the skin friction distribution.

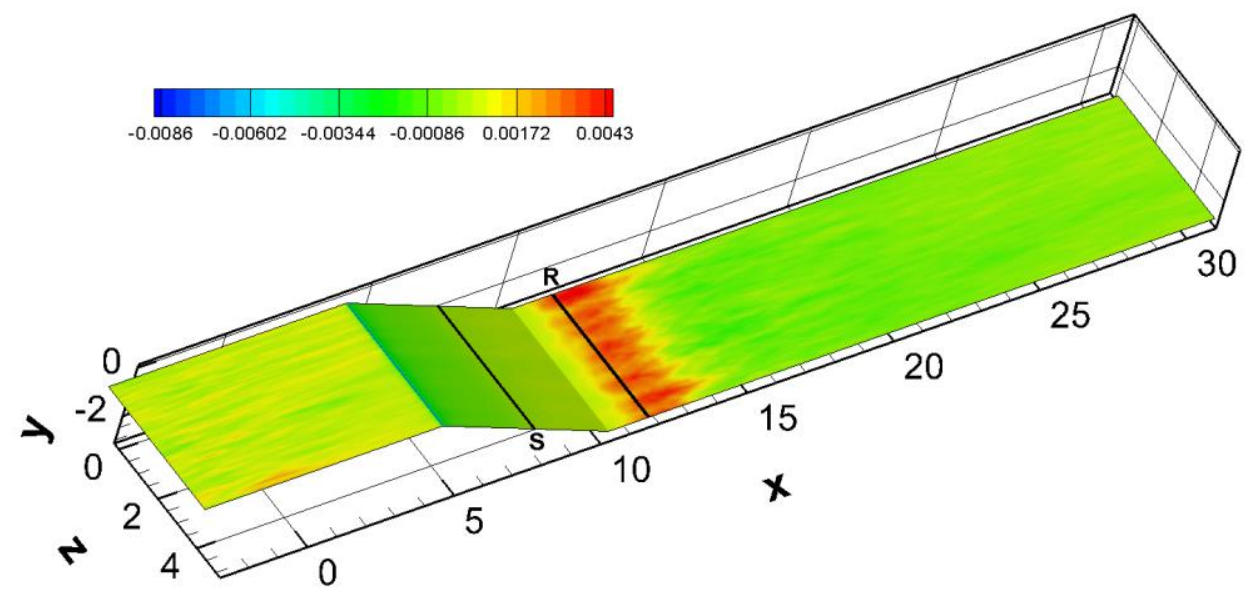

FIG. 15. Time averaged Stanton number contour for Case 2.

\section{Mean profiles}


The evolutions of velocity and temperature profiles are analysed in this section. Only results from Case 2 will be presented hereafter unless otherwise stated. The mean velocity profiles $\left\langle u_{S}\right\rangle$ extracted from predefined 13 sample lines shown in FIG. 16 are presented in FIG. 17, in which $d$ denotes the perpendicular distance to the wall surface, $\left\langle u_{S}\right\rangle$ is the mean velocity perpendicular to sample lines, and $x_{W}$ is the $x$-coordinate of the foot of the sample line.

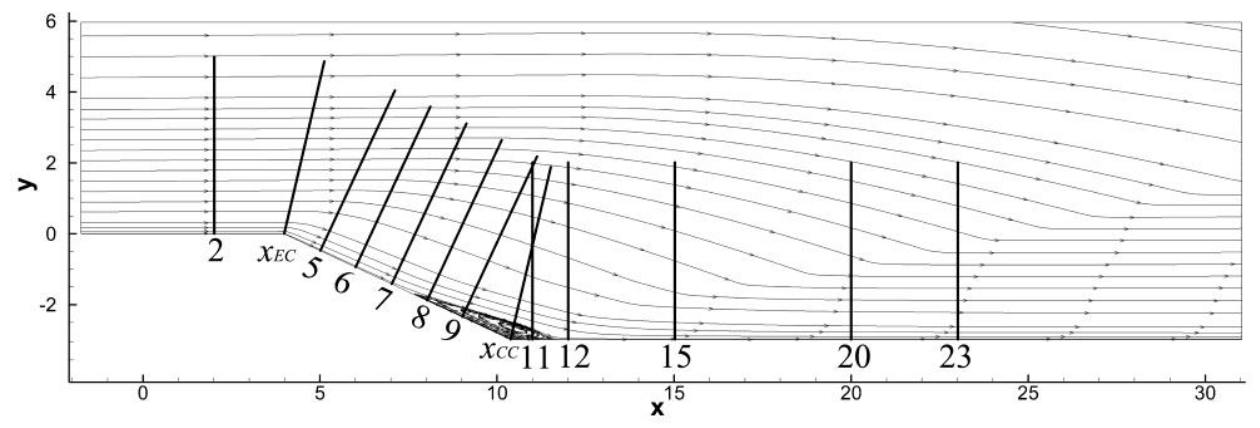

FIG. 16. Sampling lines in the $x-y$ plane.

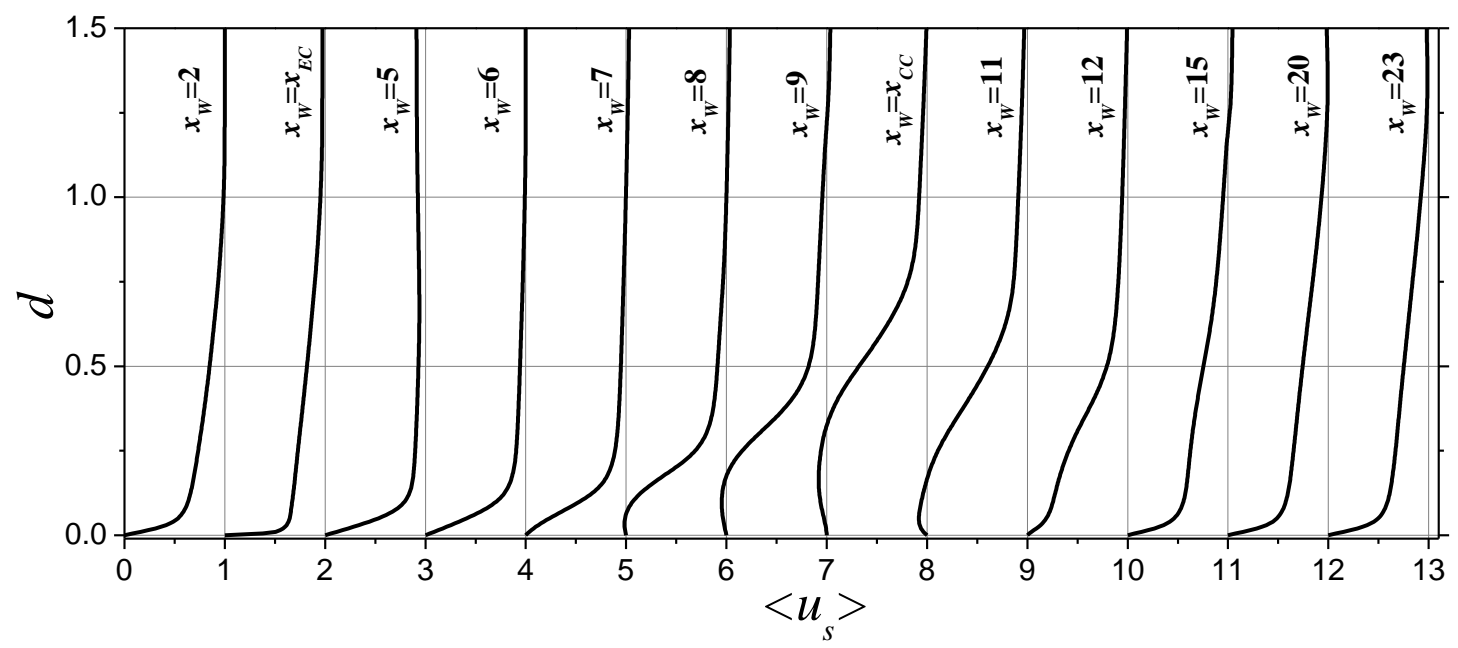

FIG. 17. The development of the mean velocity profiles. Each profile is shifted 1 unit along the horizontal axis against its upstream profile.

It is found that from EC to the middle of the ramp (i.e. $x_{w}=7$ ) the inner velocity is reduced with the thickening of the inner low-speed layer, probably due to the trend towards relaminarization and the outer part of the boundary layer being accelerated during the expansion process. At $x_{W}=8$, the negative value of $\left\langle u_{S}\right\rangle$ can be observed, indicating the appearance of the reverse flow. The height of the separation bubble increases and reaches the maximum at CC. After the reattachment, the boundary layer redevelops and finally recovers close to an equilibrium state near the exit of the computational domain, where the velocity profile is similar to that of the upstream undisturbed boundary layer.

The velocity profiles in local wall units $u_{v d}^{+}$upstream and downstream of the separation bubble are plotted in FIG. 18 (a) and (b) respectively. As we can see from FIG. 18 (a) that from $x_{W}=2$ to $x_{W}=x_{E C}$, the velocity profile shifts downward dramatically, which is caused by the great increase of the local friction velocity $u_{\tau}$ due to the spike of the skin friction at EC as demonstrated in FIG. 11. At $x_{W}=5$ and $x_{W}=6$, the near-wall part of the velocity profile matches well with the linear 
law of the sub-layer $u_{v d}^{+}=y^{+}$, but somehow the log-law does not exist anymore. Instead, the velocity develops into a wakelike profile immediately above the linear sub-layer, which is in agreement with the experimental observations in expansion corner flows. ${ }^{12,13,17}$ This kind of change of the velocity profile can be explained by the suppression of turbulence structures that are responsible for the momentum transport in the log-layer during the interaction with the expansion wave. This observation is also in agreement with the two-layer model proposed by Gillis et al. ${ }^{20}$, in which the anisotropic and productive turbulence evolves into isotropic 'debris' in the outer layer. Downstream of $x_{W}=6$, the pressure gradient changes from negative values to positive value. As result, the upward shift of the log-like layer is observed in this section and the velocity profile starts to be similar with the velocity profile in the transitional zone ${ }^{47,71}$. At the edge of the separation bubble at $x_{W}=7$, due to the skin friction being close to 0 , the whole velocity moves upwards.
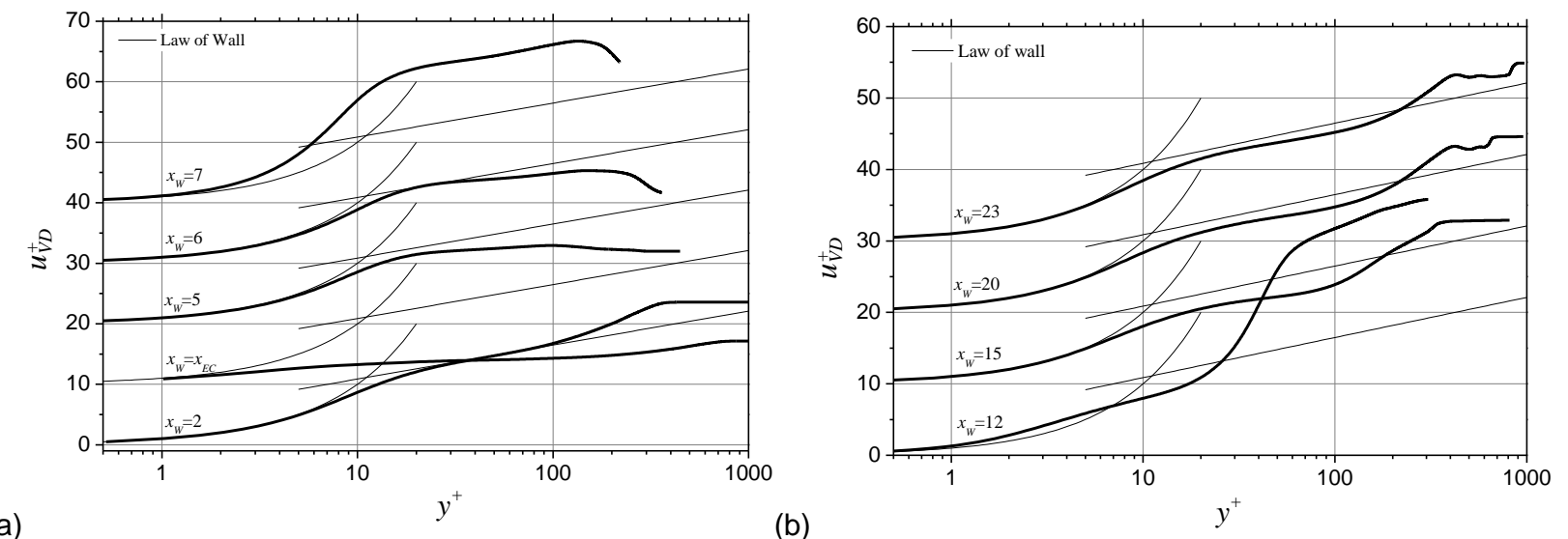

FIG. 18. The mean velocity profiles $u_{v d}^{+} \sim y^{+}$normalized with the local wall unit. (a): upstream of the separation point, (b) downstream of the reattachment point. Each profile is shifted 10 units along the vertical axis against its upstream profile.

From FIG. 18 (b) we can see the process of the recovery of the boundary layer towards the equilibrium state. Near the reattachment point $\left(x_{w}=12\right)$, the velocity profile is similar with that at the separation point, since the skin friction at both points is close to zero. As the recovery of the boundary layer, the near-wall linear layer is firstly established and the log-layer is gradually recovered towards the standard law of wall. At $x_{w}=23$, the velocity profile is close to the undisturbed profile at $x_{w}=2$, but still not fully recovered to the equilibrium state.

The mean temperature profiles at these sample lines are shown in FIG. 19. At $x_{W}=x_{E C}$, we can see the temperature in the near-wall region is reduced sharply. Further downstream, the temperature in the outer part of the thermal boundary layer is consistently reduced during the expansion process; however, the thermal layer inside the separation bubble begins to thicken. Furthermore, the temperature in the separation bubble has shown near adiabatic characteristics with small gradients in the near-wall region. The reason for this could be attributed to the lack of thermal transport due to the closed streamlines in the separation bubble and the wall heat flux causes the increase of the thickness of the thermal layer, which means the 
accumulation of interior energy of the fluid in the separation zone. Therefore, we can see the Stanton number in this region is close to zero in FIG. 12. Downstream of the separation bubble, the convection and the turbulence transport would release the reserved interior energy in the separation zone towards the wall. Consequently, the Stanton number begins to increase dramatically downstream of the reattachment point and reaches a maximum value near $x_{W}=12$. Further downstream, the temperature profile recoveries towards the shape similar to that of the incoming boundary layer.

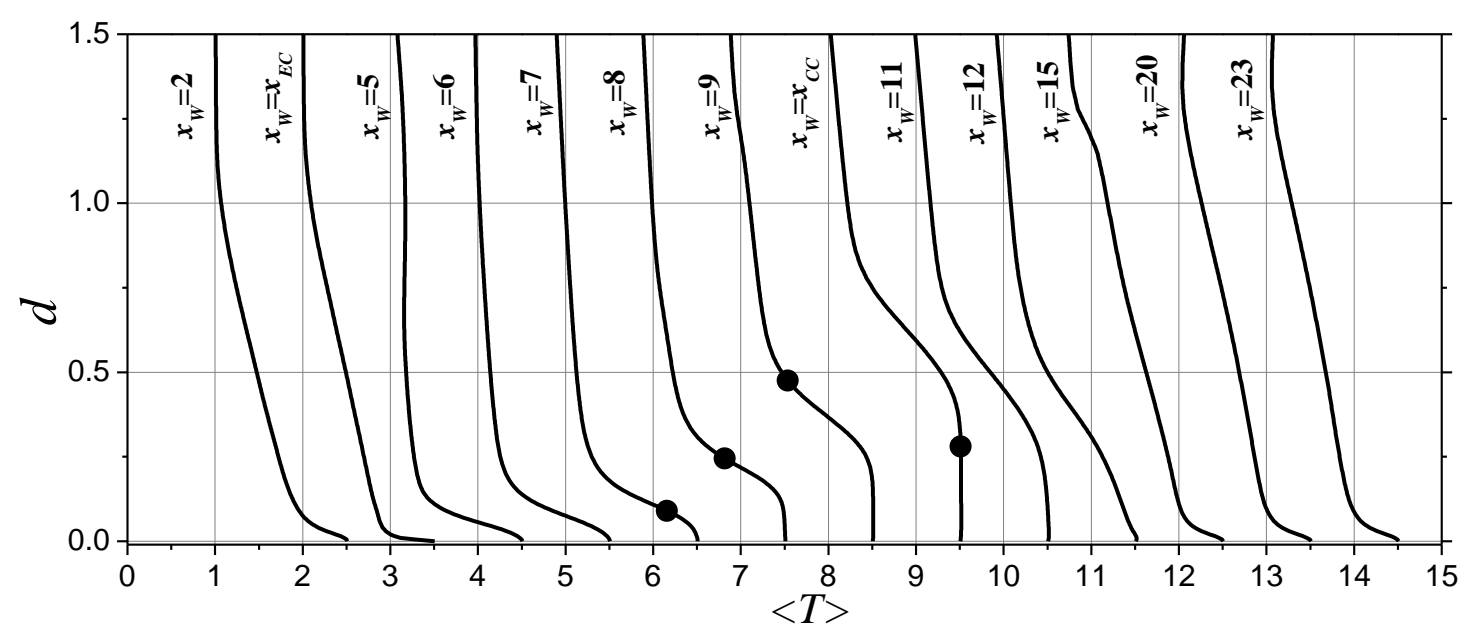

FIG. 19. The development of the mean temperature profiles. The black symbols on the profile denote the edge of the separation bubble, which is the outmost closed streamline. Each profile is shifted 1 unit along the horizontal axis against its upstream profile.

\section{Reynolds stresses}

The fields of the normal Reynolds stress components and the turbulence kinetic energy (defined as $=0.5\left\langle u_{j}^{\prime \prime} u_{j}^{\prime \prime}\right\rangle$ ) are shown in FIG. 20. During the expansion process, all components of Reynolds stresses are suppressed. Downstream of CC, however, they are all greatly amplified due to the interaction with the shock-wave. The maximum turbulence kinetic energy can be found near the reattachment point and its peak is placed in the middle of the boundary layer rather than that in the near wall region, which means the fluctuations are dominated by the turbulence in the mixing layer generated during SWTBLI, as reported in other SWTBLI flows. ${ }^{72,73}$ Further downstream, the turbulence in the outer region of the boundary layer keep damping while $\left\langle u^{\prime \prime} u^{\prime \prime}\right\rangle$ and $\left\langle w^{\prime \prime} w^{\prime \prime}\right\rangle$ begin to grow in the near-wall region, which indicates the regeneration of wall turbulence during the recovery of the boundary layer. 
(a)

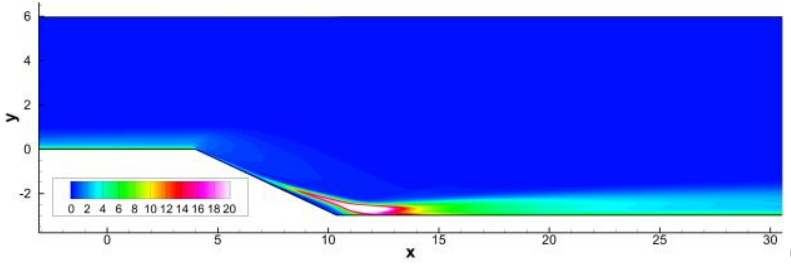

(b)

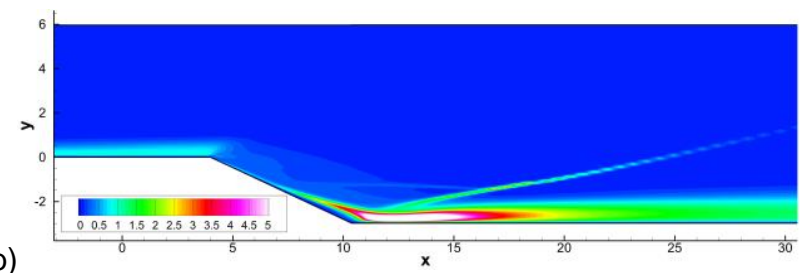

(c)

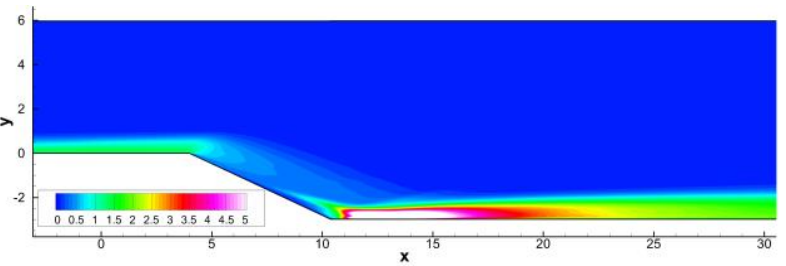

(d)

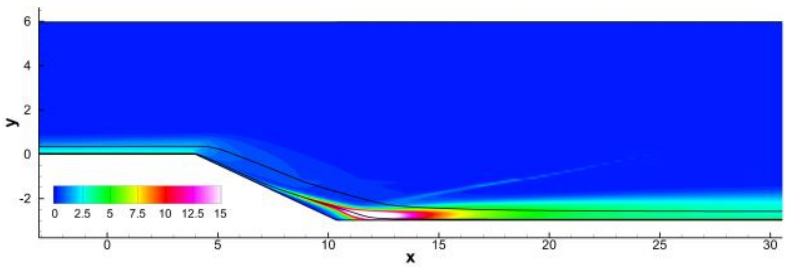

FIG. 20. Components of Reynolds normal stresses. (a): $\left\langle u^{\prime \prime} u^{\prime \prime}\right\rangle,(\mathrm{b}):\left\langle v^{\prime \prime} v^{\prime \prime}\right\rangle,(\mathrm{c}):\left\langle w^{\prime \prime} w^{\prime \prime}\right\rangle$, (d): $K$. All variables are normalized with the square of friction velocity $u_{\tau}^{2}$ at the reference place. Two streamlines are shown in (d) with black solid lines.

It is worth noting that the turbulence stresses in the ramp region present different modes of evolution in the inner layer and the outer layer regions, especially for $\left\langle u^{\prime \prime} u^{\prime \prime}\right\rangle$. Therefore a two-layer structure of turbulence can be identified in the ramp region, which is consistent with the experimental observation of Gillis et al. ${ }^{20}$ in the convexly curved boundary layer.

In the outer layer, the turbulence fluctuations are constantly suppressed along the ramp. On the other side, in the near-wall inner layer, the Reynolds stresses are reduced significantly only in a small region near EC, and then they begin to increase after the EC location. Therefore, the differences between the inner and the outer layers become more and more distinguishable during the evolution along the ramp region.

To further investigate this property, the evolution of Reynolds stresses along two streamlines is analysed. One streamline is in the near-wall region (S1: traced from the coordinate: $(x=0, y=0.03))$ and the other one is located in the middle of the boundary layer (S2: traced from the coordinate: $(x=0, y=0.35)$ ), as plotted in FIG. 20 (d). The evolutions of the normal components of the Reynolds stresses and turbulence kinetic energy along the two streamlines are plotted in FIG. 21. Along the streamline $\mathrm{S} 1,\left\langle u^{\prime \prime} u^{\prime \prime}\right\rangle$ and $\left\langle w^{\prime \prime} w^{\prime \prime}\right\rangle$ have shown significant reductions within a shorter distance passing through EC, whilst $\left\langle v^{\prime \prime} v^{\prime \prime}\right\rangle$ has only a negligible oscillation around EC position. Downstream of EC, all components of the Reynolds stress increase along the streamline. The rate of increase is then largely amplified after the interaction with the front shockwave at $x \approx 7$, and the rate of increase is further amplified after the interaction with the rear shock-wave at CC location. The peak of the normal Reynolds stresses can be found near the reattachment point.

In contrast, along the streamline S2, all components are gradually reduced downstream EC, until the streamline entering the mixing layer formed during SWTBLI, and then the increase of all components of Reynolds stresses can be seen. Therefore, it is clear that the turbulence in the inner layer and the outer layer has undergone a completely different evolution 
process, which implies the different turbulence suppression and re-development mechanisms in the inner and the outer layers along the ramp wall surface.

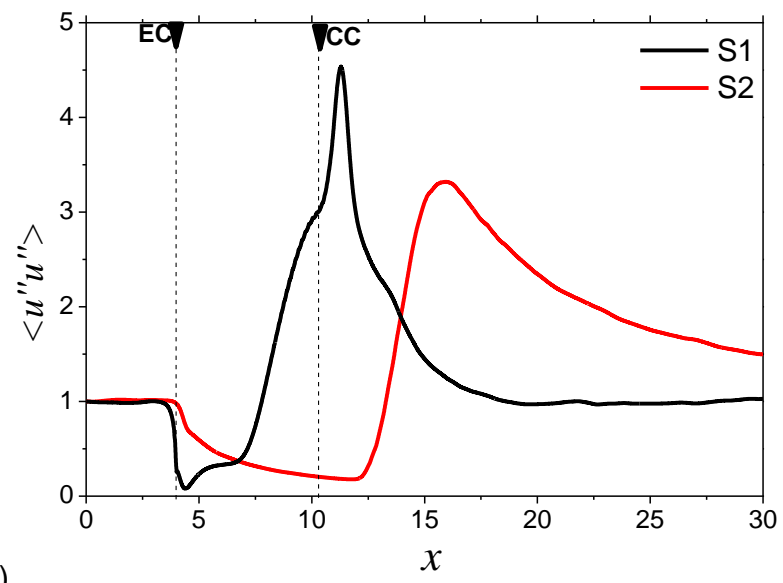

(a)

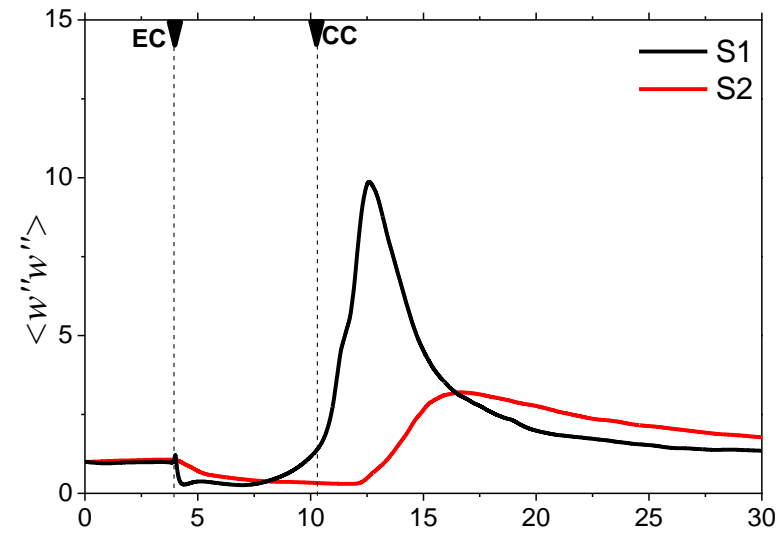

(c)

$x$

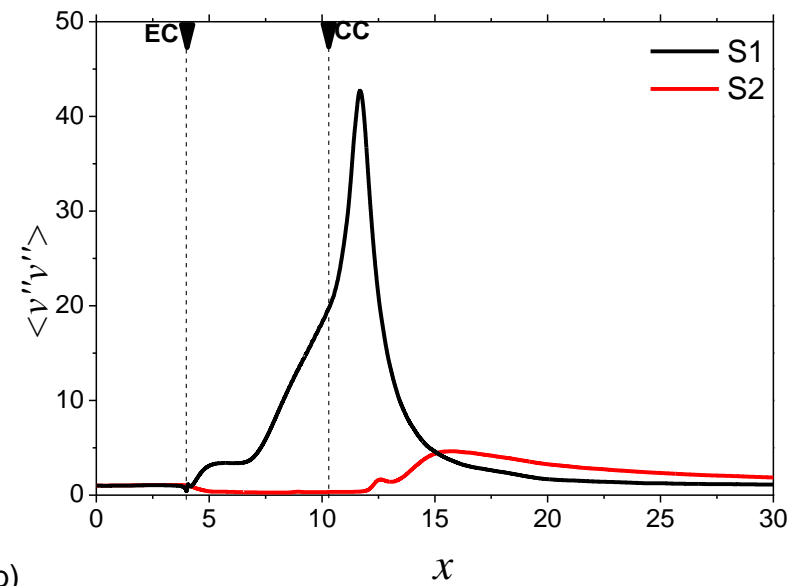

(b)

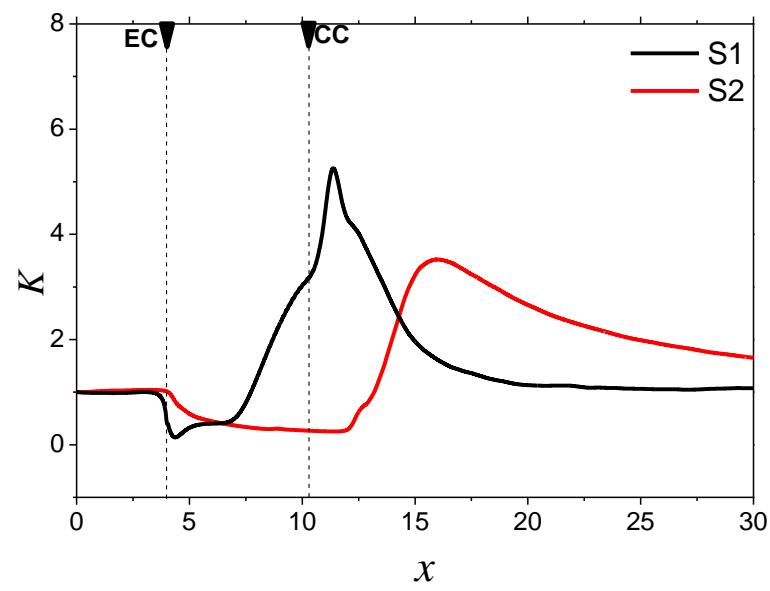

FIG. 21. Evolution of (a): $\left\langle u^{\prime \prime} u^{\prime \prime}\right\rangle$, (b): $\left\langle v^{\prime \prime} v^{\prime \prime}\right\rangle,(\mathrm{c}):\left\langle w^{\prime \prime} w^{\prime \prime}\right\rangle$, and (d): $K$, along the streamlines S1 and S2. All variables are normalized with their values at the reference plane.

The contours of the Reynolds shear stress $\left\langle u^{\prime \prime} v^{\prime \prime}\right\rangle$ and its evolution along two streamlines S1 and S2 are shown in FIG. 22 and FIG. 23, respectively, from which a similar trend of the different evolution processes in the inner and the outer layers to the normal Reynolds stresses can be seen. In FIG. 23 (b), the cross correlation of the Reynolds shear stress $R\left(u^{\prime \prime}, v^{\prime \prime}\right)=$ $\left\langle u^{\prime \prime} v^{\prime \prime}\right\rangle / \sqrt{\left\langle u^{\prime \prime} u^{\prime \prime}\right\rangle\left\langle v^{\prime \prime} v^{\prime \prime}\right\rangle}$ is plotted to study the property of turbulence structures. As pointed by Kim et al. ${ }^{74}$ in wall turbulence that $R\left(u^{\prime \prime}, v^{\prime \prime}\right)$ represents the existence of certain organized turbulent motions. According to FIG. 23 (b), we can see $R\left(u^{\prime \prime}, v^{\prime \prime}\right)$ along S1 and S2 evolve in very different ways. Along the streamline $\mathrm{S} 1, R\left(u^{\prime \prime}, v^{\prime \prime}\right)$ is increased more than two times its upstream value, which means the coherence or anisotropy of turbulent motions is obviously strengthened. On the contrary, $R\left(u^{\prime \prime}, v^{\prime \prime}\right)$ is reduced to almost zero along the streamline $\mathrm{S} 2$, indicating that the turbulence is almost isotropic. In 
the two-layer model of Gillis et al. ${ }^{20}$, the similar characteristic of the structural parameters in the inner and the outer layers was reported.

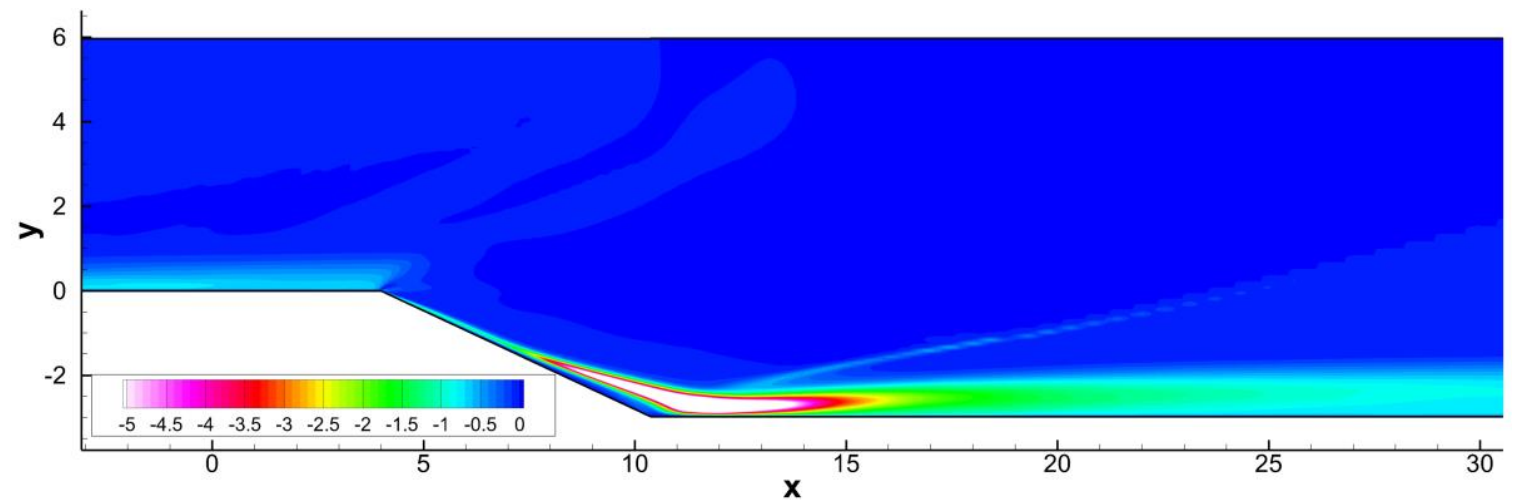

FIG. 22. Reynolds shear stresses normalized with $u_{\tau}^{2}$ at the reference place.

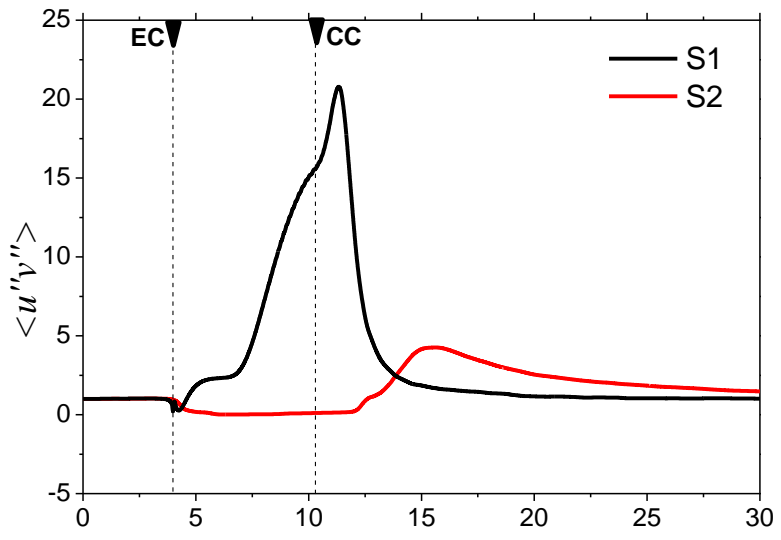

(a)

FIG. 23. Evolutions of the Reynolds shear stress (a) and its cross correlation (b) along the streamlines S1 and S2. All variables are normalized with their values at the reference plane.

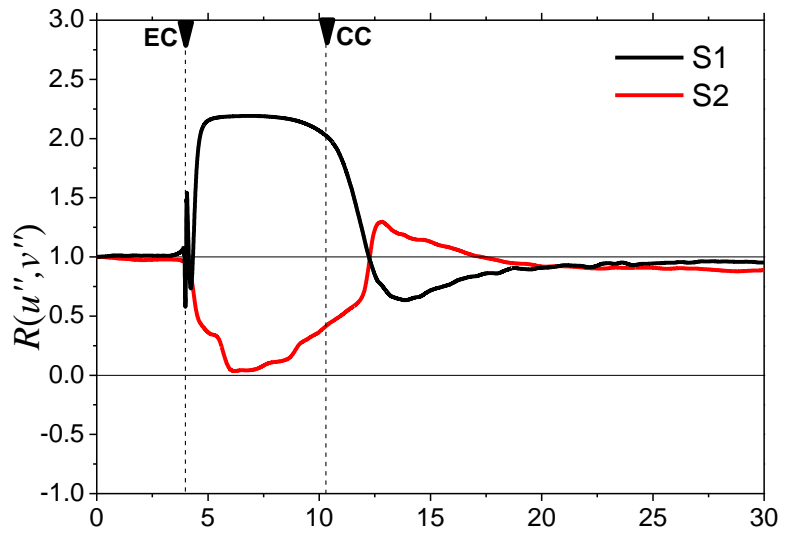

$x$

The peak values of the Reynolds stresses and turbulence kinetic energy and their distances to the wall along the streamwise $x$-direction are extracted and shown in FIG. 24. We can see the great amplification of the peak values of all components due to the interaction with the shock-wave.

The peaks of all components of Reynolds stresses are located in the inner layer upstream of EC, and the peak of $\left\langle u^{\prime \prime} u^{\prime \prime}\right\rangle$ is the closest to the wall, which is a typical characteristic of the wall turbulence. In the ramp region, the peaks of all components grow around the edge of the separation bubble. After the CC location, different components of Reynolds stresses present different evolution processes. The peak of $\left\langle w^{\prime \prime} w^{\prime \prime}\right\rangle$ moves to the near wall region, and then almost immediately after the reattachment point, it gradually re-develops outwards. This implies the existence of certain turbulence structures downstream of $\mathrm{CC}$, which contributes greatly to $\left\langle w^{\prime \prime} w^{\prime \prime}\right\rangle$ in the near-wall region. One type of the turbulence structures could be the Görtler vortices according to the previous analysis. Downstream of CC, the peak of $\left\langle u^{\prime \prime} u^{\prime \prime}\right\rangle$ firstly occur around $d_{\text {Peak }}=0.3$, where the core of the mixing layer is located. Similar to that of $\left\langle w^{\prime \prime} w^{\prime \prime}\right\rangle$, the peak of $\left\langle u^{\prime \prime} u^{\prime \prime}\right\rangle$ also shifts to the near-wall 
region and stays there further downstream, which means the attached wall turbulence is regenerated in the near-wall region. The process of the regeneration of wall turbulence is within a shorter distance than that in the interaction of an oblique shockwave impinging onto a flat plate boundary layer ${ }^{60}$, which could be again attributed to the strong flow fluctuations induced by the Görtler vortices. The peaks of $\left\langle v^{\prime \prime} v^{\prime \prime}\right\rangle$ and $\left\langle u^{\prime \prime} v^{\prime \prime}\right\rangle$ are located in the outer part of the boundary layer, and move further away from the wall, indicating that they are dominated by the free shear of the mixing layer. Downstream of CC, the peak of turbulence kinetic energy $K$ is firstly dominated by $\left\langle v^{\prime \prime} v^{\prime \prime}\right\rangle$ in the core of the mixing layer, and then with the development of the regenerated wall turbulence, the peak of $K$ returns to the near-wall region where the peak of $\left\langle u^{\prime \prime} u^{\prime \prime}\right\rangle$ locates.
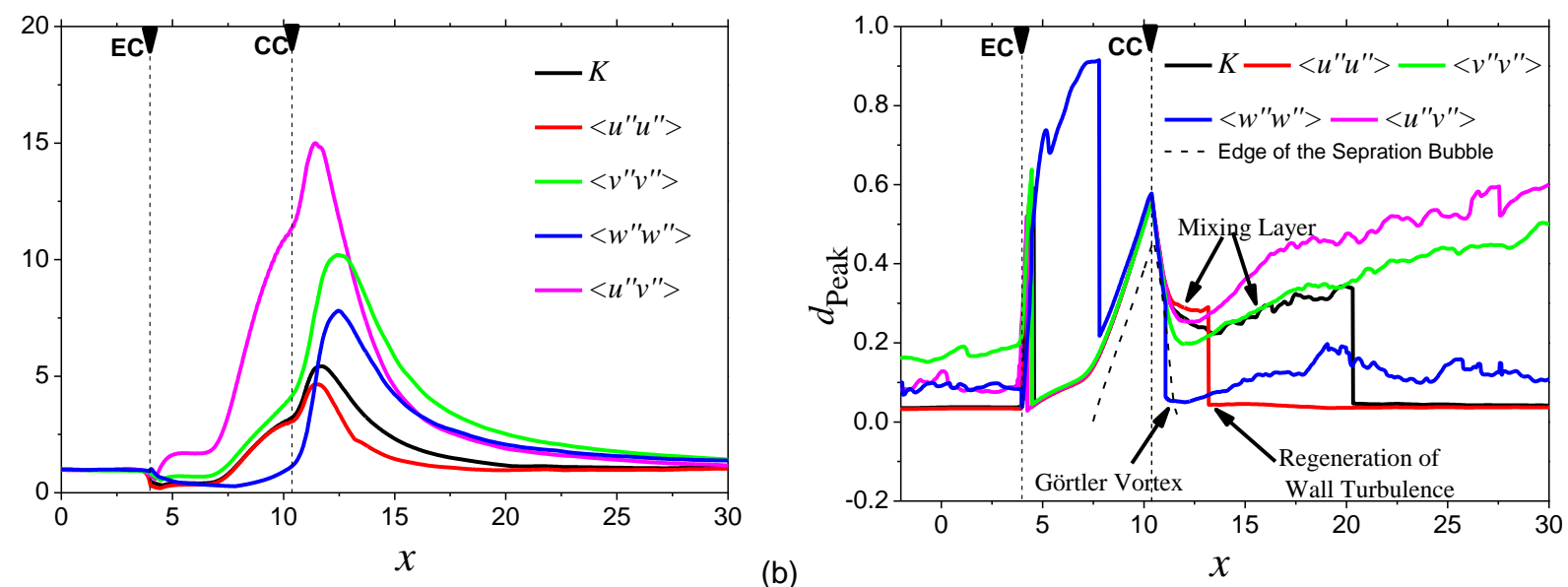

(a)

(b)

FIG. 24. Distributions of the peak values (a) and their distances (b) to the wall surface. The peak values are normalized with their values at the reference location.

The Reynolds stresses profiles along the 13 pre-defined sample lines are further studied. To better describe the Reynolds stresses above the slant ramp surface, the Reynolds stresses are decomposed according to the directions parallel and normal to the surface of the ramp respectively, which is expressed as

$$
\begin{aligned}
& \left\langle u_{s}^{\prime \prime} u_{s}^{\prime \prime}\right\rangle=\left\langle\left(\vec{u}^{\prime \prime} \cdot \overrightarrow{n_{s}}\right)\left(\vec{u}^{\prime \prime} \cdot \overrightarrow{n_{s}}\right)\right\rangle \\
& \left\langle u_{n}^{\prime \prime} u_{n}^{\prime \prime}\right\rangle=\left\langle\left(\vec{u}^{\prime \prime} \cdot \overrightarrow{n_{n}}\right)\left(\vec{u}^{\prime \prime} \cdot \overrightarrow{n_{n}}\right)\right\rangle \\
& \left\langle u_{s}^{\prime \prime} u_{n}^{\prime \prime}\right\rangle=\left\langle\left(\vec{u}^{\prime \prime} \cdot \overrightarrow{n_{n}}\right)\left(\vec{u}^{\prime \prime} \cdot \overrightarrow{n_{s}}\right)\right\rangle
\end{aligned}
$$

in which $\vec{u}^{\prime \prime}$ is the fluctuation velocity vector, $\overrightarrow{n_{s}}$ and $\overrightarrow{n_{n}}$ are the unit vector parallel and normal to the surface of the wall, respectively.

FIG. 25 shows the normal and the shear components of the Reynolds stress profiles along sample lines. From FIG. 25 (a), the suppression of $\left\langle u_{s}^{\prime \prime} u_{s}^{\prime \prime}\right\rangle$ in the outer layer and its growth in the inner layer can be seen from $x_{W}=2$ to the CC location, due to the two-layer structure of the flow along the ramp. The near-wall peaks are exhibited in the $\left\langle u_{s}^{\prime \prime} u_{s}^{\prime \prime}\right\rangle$ profiles, and the position of the peak gradually moves away from the ramp surface, which can be attributed to the increase of the height of the separation bubble. The peak reaches the highest position at CC. At $x_{W}=12$ where the flow is reattached, a double-peak 
structure of the $\left\langle u_{s}^{\prime \prime} u_{s}^{\prime \prime}\right\rangle$ profile develops, in which the outer peak is from the upstream turbulence and enhanced due the SWTBLI effect and the inner peak is the newly generated near-wall turbulence due to the flow reattachment. Further downstream, the outer peak decreases due to the decay of the mixing layer generated during SWTBLI and the near-wall peak keeps growing within the process of the recovery of the boundary layer.

It is interesting to note that there are two types of two-layer structures appearing in the expansion-compression flow. The first one develops during the expansion process consisting of an outer layer with decaying almost isotropic turbulence and an inner layer with anisotropic productive turbulence, as discussed in detail above. The second one develops after the flow reattachment, in which the fluctuations in the outer layer are dominated by the free-shear turbulence in the mixing layer due to SWTBLI and the inner layer is the newly regenerated wall turbulence. Furthermore, the turbulence in the inner layer of the first type of the two-layer structures also interacts with the mixing layer in the second and has a great contribution to the strong fluctuations in the mixing layer. Therefore, the following scenario can be described based on the analysis above,

1. The equilibrium boundary layer passes through EC and interacts with the expansion fan. During this process, the turbulence in the outer layer is consistently supressed by the expansion wave and evolves towards isotropic turbulence, but the turbulence in the near-wall inner layer preserves the coherence of the wall turbulence and gets amplified along the edge of the separation bubble. Therefore, the first two-layer structure develops.

2. The disturbed boundary layer interacts with the shock-wave. During this process, the turbulence in the outer layer is very weak, therefore nothing important happens. The turbulence in the inner layer gets even stronger during the interaction with the shock-wave and contributes greatly to the turbulence fluctuations in the mixing layer generated during SWTBLI.

3. After the flow reattachment, the interaction of highly fluctuant flow with the wall will cause the regeneration of wall turbulence and the wall turbulence gets stronger during the recovery process. Meanwhile, the mixing layer diffuses and decays gradually. Therefore, another two-layer structure is formed, in which the outer layer is the decaying mixing layer and the inner layer is the newly generated wall turbulence. 


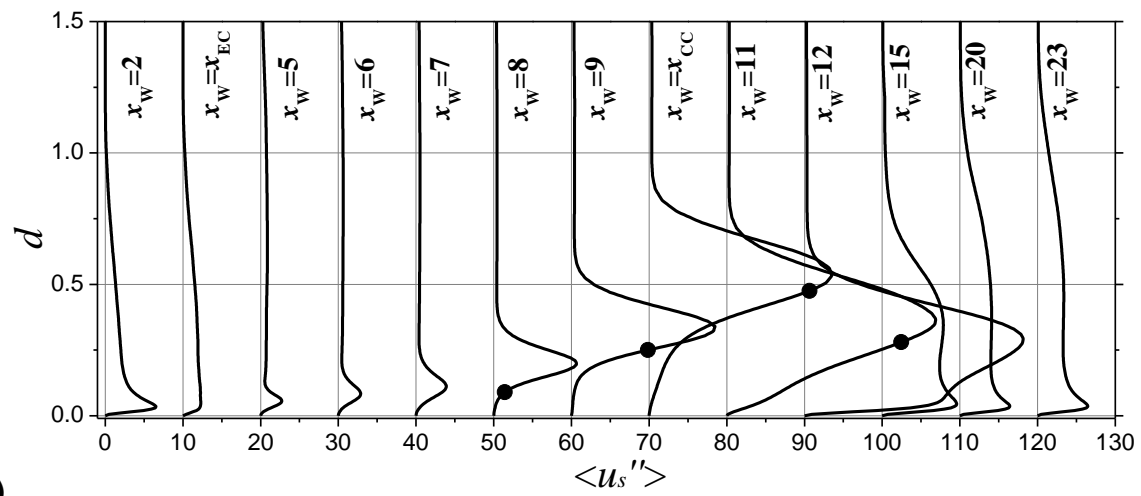

(a)

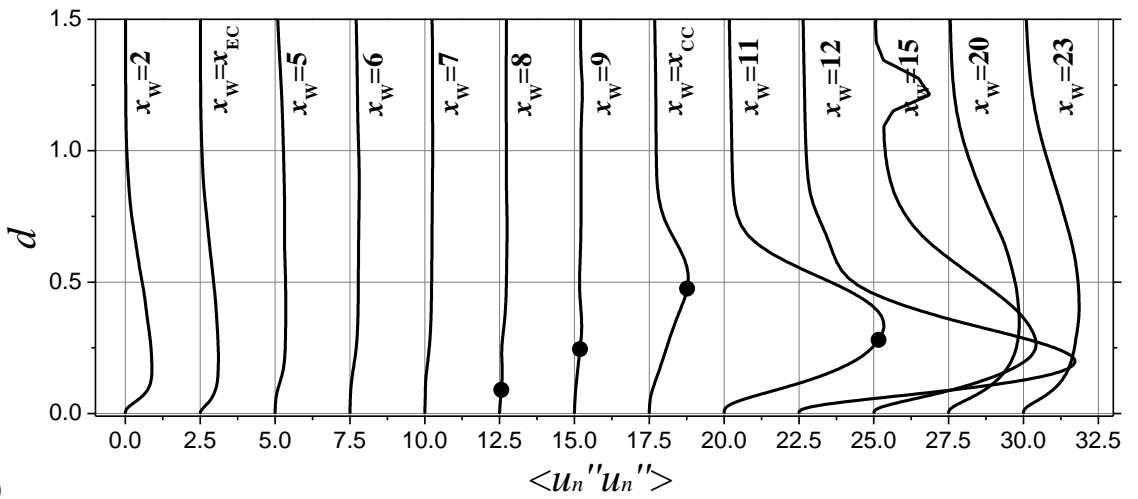

(b)

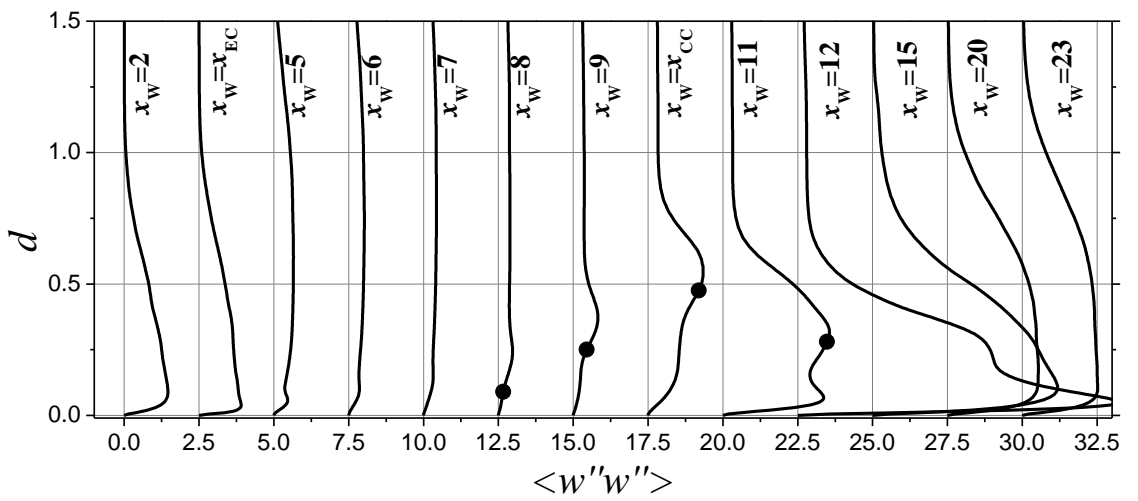

(c)

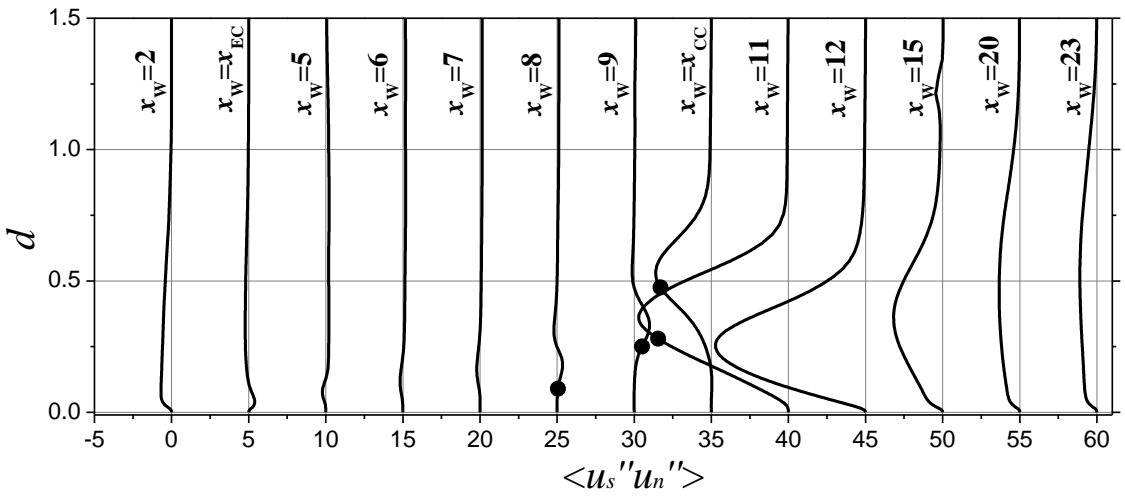

(d)

FIG. 25. Profiles of(a): $\left\langle u_{s}^{\prime \prime} u_{s}^{\prime \prime}\right\rangle,(\mathrm{b}):\left\langle u_{n}^{\prime \prime} u_{n}^{\prime \prime}\right\rangle,(\mathrm{c}):\left\langle w^{\prime \prime} w^{\prime \prime}\right\rangle$ and (d): $\left\langle u_{s}^{\prime \prime} u_{n}^{\prime \prime}\right\rangle$ along the sample lines. The black symbols on the profile denote the edge of the separation bubble. Each profile is shifted 10, 2.5, 2.5 and 5 units along the horizontal axis in (a), (b), (c) and (d) against its upstream profile respectively. 
From profiles of $\left\langle u_{n}^{\prime \prime} u_{n}^{\prime \prime}\right\rangle,\left\langle w^{\prime \prime} w^{\prime \prime}\right\rangle$ and $\left\langle u_{s}^{\prime \prime} u_{n}^{\prime \prime}\right\rangle$, we can also identify the development of peaks attaching the edge of the separation bubble. Inside the separation bubble, the values of Reynolds stresses are relatively low, due to 'quiet' fluid motions inside the separation region.

Beside the two two-layer structures described above, it is interesting to note that, $\left\langle w^{\prime \prime} w^{\prime \prime}\right\rangle$ develops a local peak inside of the separation bubble at $x_{W}=9$ (also can be found in FIG. 24 (b)). Therefore, there is a two-peak structure for $\left\langle w^{\prime \prime} w^{\prime \prime}\right\rangle$ upstream of the flow reattachment, in which the outer peak attaches to the edge of the separation bubble, just like the peak of $\left\langle u^{\prime \prime} u^{\prime \prime}\right\rangle$ at the same location, the other peak is in the near-wall region, which grows faster than the outer peak. At $x_{W}=11$ these two peaks have similar values, while at $x_{W}=12$, the near-wall peak becomes almost twice that of the outer peak. To study this double-peak phenomenon, the traces of local peaks of both $\left\langle u^{\prime \prime} u^{\prime \prime}\right\rangle$ and $\left\langle w^{\prime \prime} w^{\prime \prime}\right\rangle$ are plotted in FIG. 26, from which we can see that both $\left\langle u^{\prime \prime} u^{\prime \prime}\right\rangle$ and $\left\langle w^{\prime \prime} w^{\prime \prime}\right\rangle$ present double-peak structures, but their origins are rather different, which indicates different mechanisms for these two components. For $\left\langle u^{\prime \prime} u^{\prime \prime}\right\rangle$, the double-peak happens downstream of the reattachment point, which is described above in detail and also reported previously in downstream region of an impinging shock-wave/flat plate boundary layer interactions ${ }^{59,75}$. The inner peak of the double-peak structures of $\left\langle w^{\prime \prime} w^{\prime \prime}\right\rangle$ is firstly observed inside of the separation bubble, and it is quite weak compared to the outer peak. With the flow development, the inner peak develops very fast and it gets a greater strength than the outer peak near the reattachment point (FIG. 25 (c)). Downstream of the reattachment point, the inner and the outer peaks merge together in the near-wall region. The double-peak structure of $\left\langle w^{\prime \prime} w^{\prime \prime}\right\rangle$ is connected with a 3D large-scale structure in the separation bubble, which is a kind of steady vortex structure, since the flow inside the separation bubble is less fluctuant. This 3D structure should be the Görtler-type vortex, and its rotation in the streamwise direction has great contributions to the spanwise velocity fluctuations. Downstream, the two peaks of $\left\langle w^{\prime \prime} w^{\prime \prime}\right\rangle$ have merged into one near-wall peak, and the traces of peaks of $\left\langle w^{\prime \prime} w^{\prime \prime}\right\rangle$ and $\left\langle u^{\prime \prime} u^{\prime \prime}\right\rangle$ collapse after $x_{W}=12$, which indicates the regeneration of the wall turbulence is promoted by the $3 \mathrm{D}$ large-scale structures.

To obtain further insights into the amplification of the Reynolds shear stress, which is strongly related to the momentum transport inside the boundary layer, the cross correlation $R\left(u_{s}^{\prime \prime}, u_{n}^{\prime \prime}\right)=-\left\langle u_{s}^{\prime \prime} u_{n}^{\prime \prime}\right\rangle / \sqrt{\left\langle u_{s}^{\prime \prime} u_{s}^{\prime \prime}\right\rangle\left\langle u_{n}^{\prime \prime} u_{n}^{\prime \prime}\right\rangle}$ is calculated and shown in FIG. 27. In the upstream boundary layer at $x_{W}=2, R\left(u_{s}^{\prime \prime}, u_{n}^{\prime \prime}\right)$ exhibits a near-wall peak of $R\left(u_{s}^{\prime \prime}, u_{n}^{\prime \prime}\right)=0.46$, which is attributed to certain organized motions in the wall region ${ }^{74}$, as mentioned above. At EC, $R\left(u_{s}^{\prime \prime}, u_{n}^{\prime \prime}\right)$ is largely reduced and the peak of the correlation becomes very weak. After EC, a new peak is formed, and it keeps shifting away from the surface of the ramp in the downstream region. By marking these peak locations on the instantaneous density schlieren shown in FIG. 28, we can see that these peaks are correlated to organized turbulence motions along the ramp surface. These turbulence motions, which originate from the upstream undisturbed boundary layer, occur at the edge of the separation bubble around the 
compression corner region. Their strength is weakened with the increase of their scales, although their coherence pattern is still preserved.

(a)

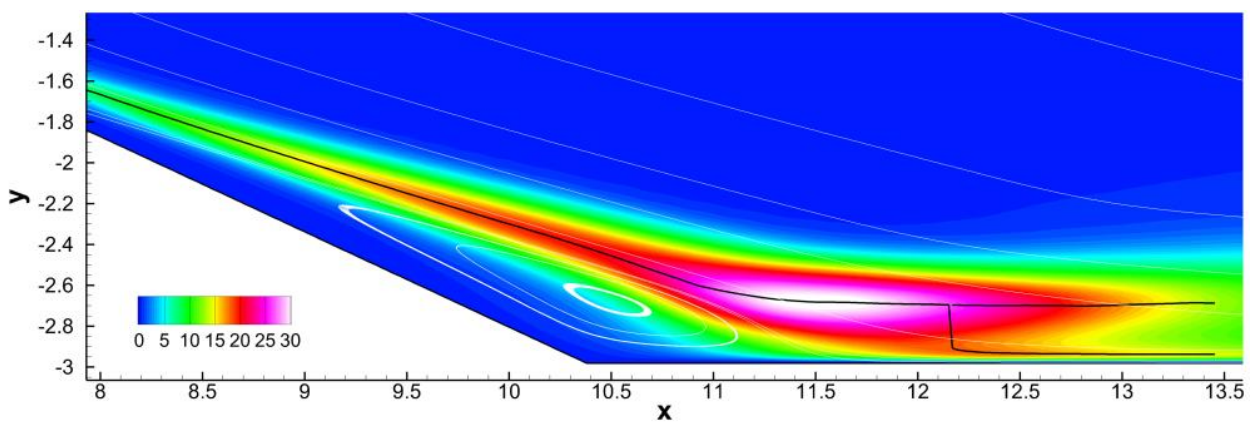

(b)

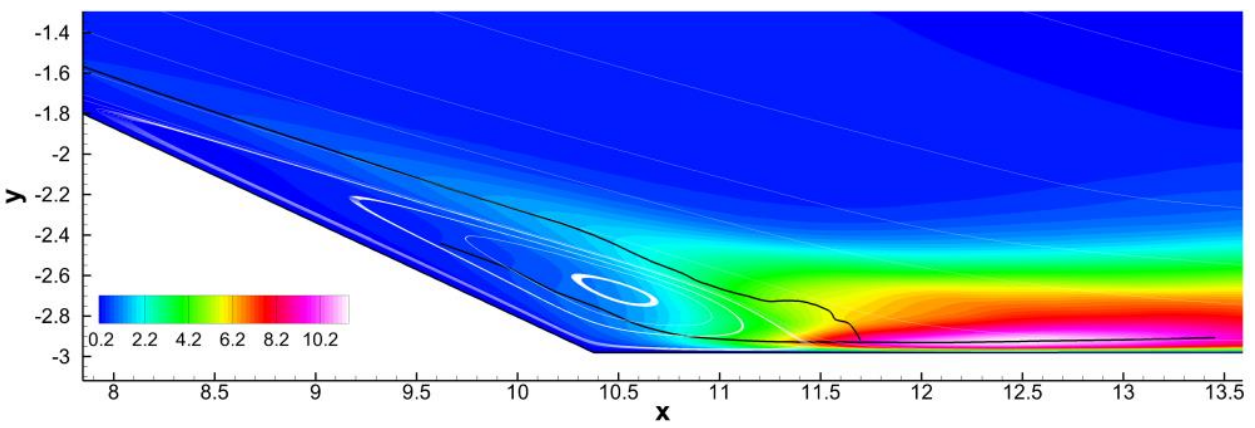

FIG. 26. Distributions of (a): $\left\langle u^{\prime \prime} u^{\prime \prime}\right\rangle$ and (b): $\left\langle w^{\prime \prime} w^{\prime \prime}\right\rangle$ in the CC region. The black thick lines denote the local peaks of the Reynolds stresses and the white thin lines denote the mean streamlines.

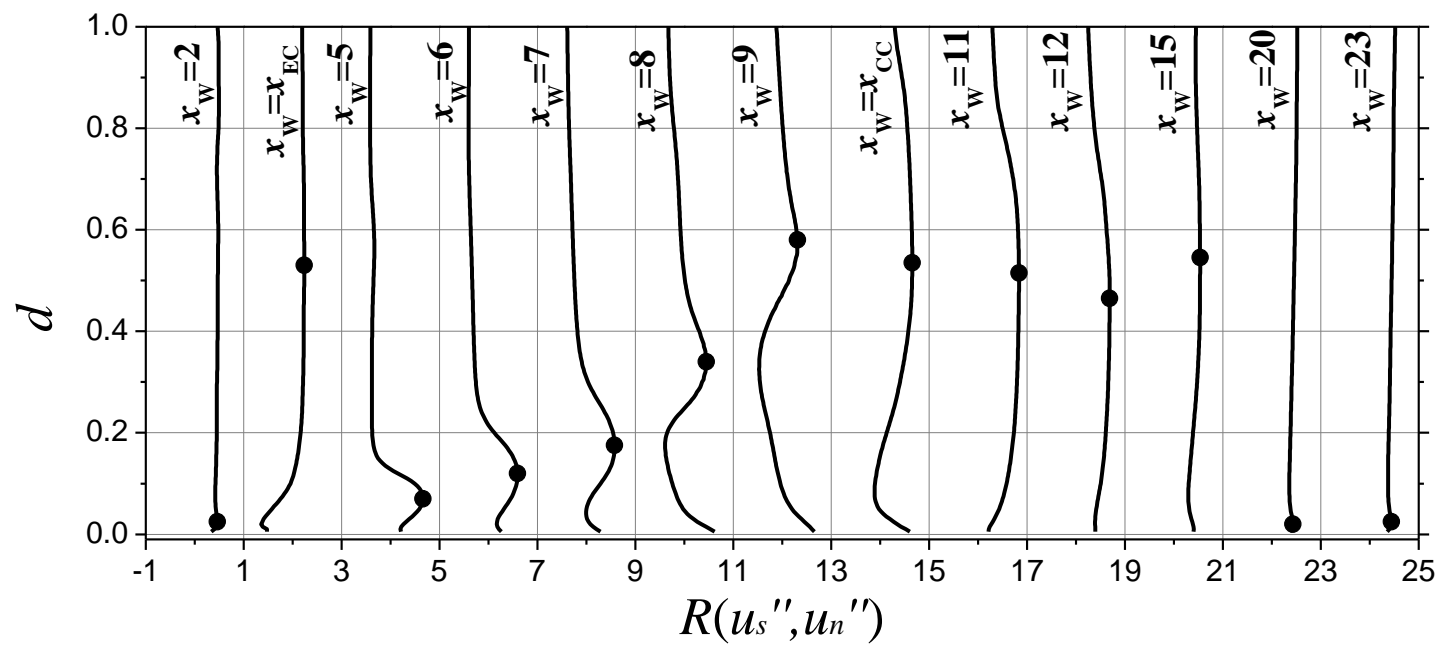

FIG. 27. The profiles of the cross correlation along the sample lines. The symbols on the profile denote the peak locations. Each profile is shifted 2 units along the horizontal axis against its upstream profile. 


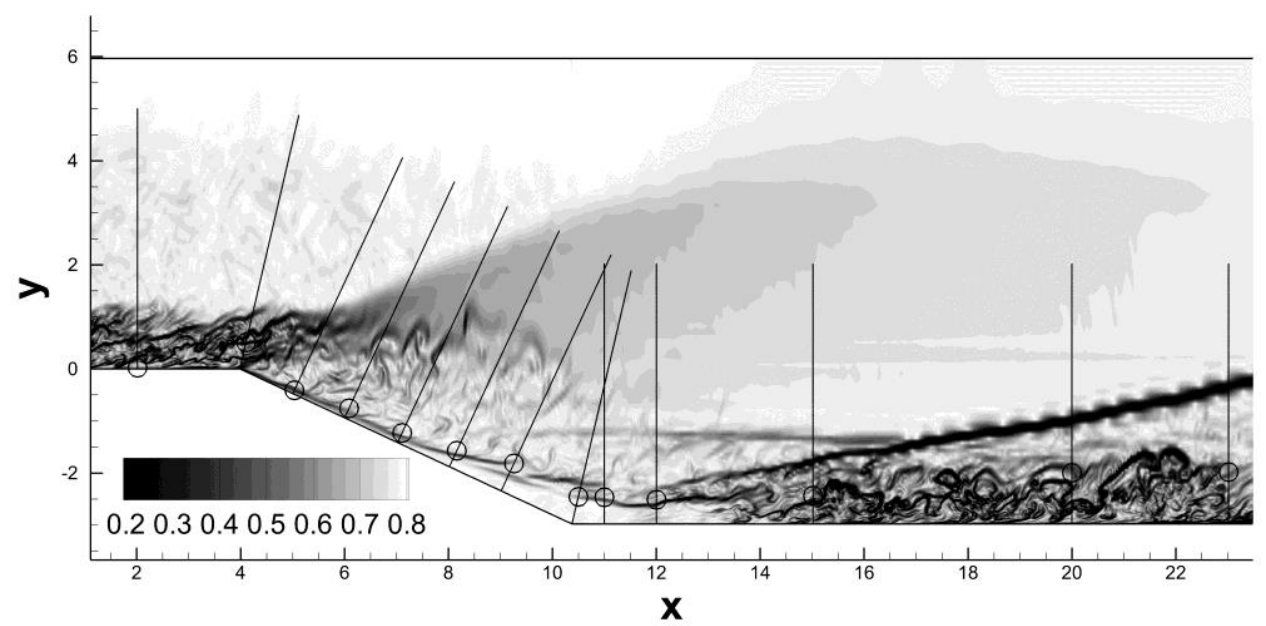

FIG. 28. The distribution of the peak of $R\left(u_{s}^{\prime \prime} u_{n}^{\prime \prime}\right)$ in the instantaneous schlieren. The peak locations are marked with open circles.

After interaction with the shock-wave, the schlieren picture shows a greater density gradient, which indicates the amplification of turbulence motions and the decrease of their scales. Downstream of the interaction region, the peaks are located in the middle of the boundary layer, which is attributed to the large-scale turbulence structures in the mixing layer.

\section{E. Instantaneous turbulence structures}

The instantaneous turbulent flow field from Case 2 has been analysed to investigate the turbulence structures. Firstly, the streamwise velocity fluctuation $u_{s}^{\prime \prime}$ along two planes containing streamlines S1 and S2 respectively are shown in FIG. 29. For the S1 plane, which locates in the near-wall region, we can see the classic streamwise elongated streaks occur in the upstream undisturbed boundary layer region and the distance between two neighbouring low-speed streaks is about $\Delta z^{+}=100$. Downstream of EC, the strength of velocity fluctuations is weakened. However, the streaky structures are still preserved and their scale is largely increased. From the second half of the ramp, we can see large-scale streaks with a spanwise length scale of about $\delta_{r e f}$ and a streamwise length scale of about $5 \delta_{r e f}$. Downstream of CC, the large-scale streaks still dominate the near-wall turbulence but they become unstable and break down to small-scale structures at about $x=15$. Further downstream, the classic streaky structures as seen in the upstream undisturbed boundary layer are re-formed, which promotes the near-wall turbulence recovery towards the equilibrium state.

In contrast, the streamwise velocity fluctuation along the S2 plane presents weak and less organized structures in the upstream undisturbed boundary layer and these fluctuations are consistently suppressed over the ramp region until the flow reattachment point. After $\mathrm{x}=15$, where the streamline enters the mixing layer region, we can see some large-scale low-speed spots, which are from the turbulence coherent structures in the mixing layer. 


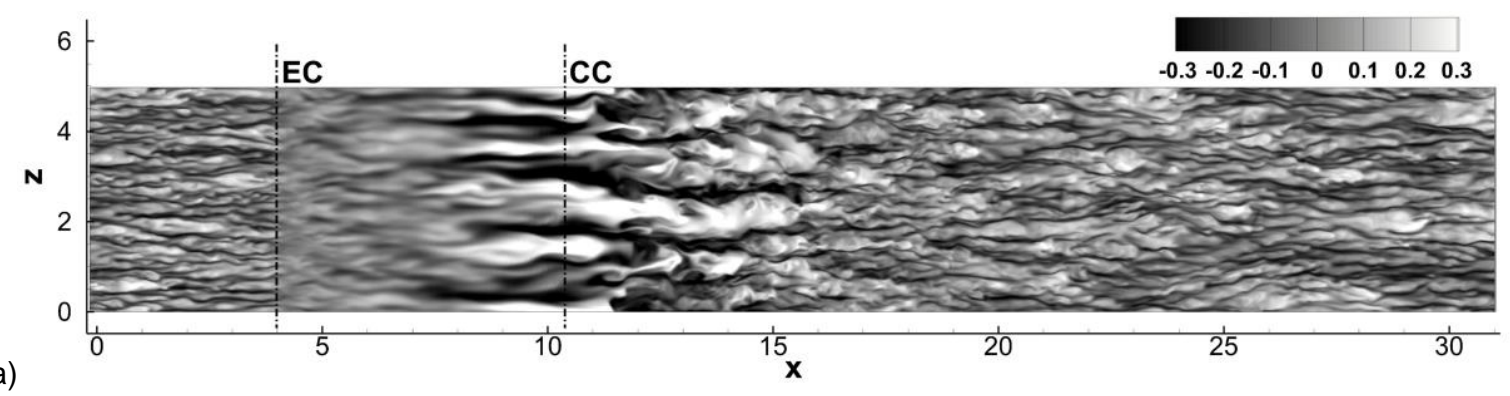

(a)

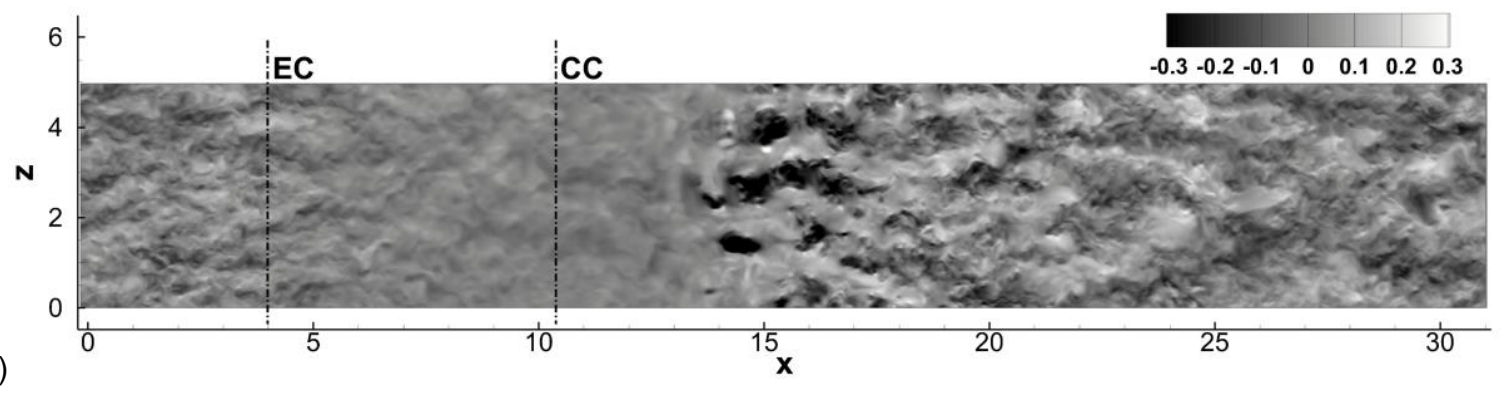

FIG. 29. Streamwise velocity fluctuation along the streamlines S1 (a) and S2 (b).

The turbulence coherent structures are further visualized with the $\lambda_{c i}$ criterion, which was proposed by Zhou et al. ${ }^{76}$ as the representation of the swirling strength. The iso-surface of $\lambda_{c i}$ coloured with the instantaneous $x$-vorticity $\omega_{x}=\frac{\partial w}{\partial y}-\frac{\partial v}{\partial z}$ is shown in FIG. 30. The coherent structures in the undisturbed boundary layer present classic streamwise elongated hairpin vortices. In the ramp region, we can rarely see any coherent structures, indicating the suppression of turbulence during the expansion process. Near the reattachment line in FIG. 30 (c), some large-scale coherent structures in the boundary layer can be identified. In the recovery region, detached structures from the mixing layer are observed. These detached large-scale structures in the outer part of the boundary layer keeps decaying along with the recovery of the boundary layer, while the hairpin structures in the near wall region are gradually regenerated during the recovery process. 


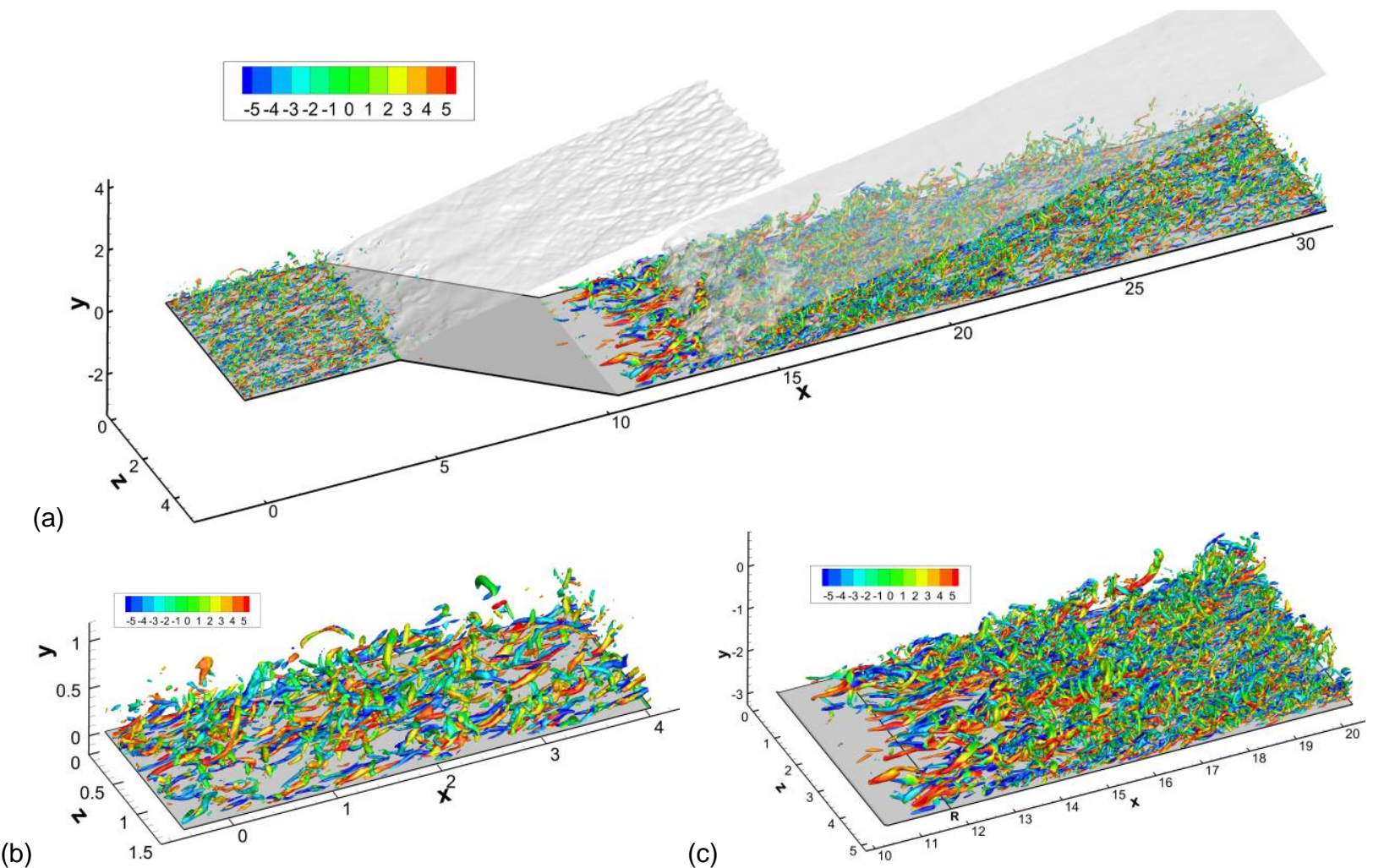

FIG. 30. Turbulence coherent structures visualized with the iso-surface of $\lambda_{c i}$ equalling to $0.8 \%$ of its global maximum and coloured with the $x$-vorticity. The expansion-wave and shock-wave are visualized by using the iso-surface of $P=0.6 P_{0}$. The structures in the undistributed boundary layer and downstream of CC region are zoomed in (b) and (c) respectively.

The enhanced visualization of turbulence coherent structures in the ramp region is shown by using the iso-surface of $\lambda_{c i}=0.16 \% \lambda_{c i, \max }$ in FIG. 31, in which the evolution of the first type of the two-layer structures along the ramp can be identified. The coherent structures from the outer part of the undisturbed boundary layer are gradually damped out with the disappearance of their coherence during the expansion process. In the inner layer however, the quasi-streamwise structures are preserved all the way along the ramp. Therefore, downstream of the separation line, the large-scale quasi-streamwise vortices can be seen very clearly. These structures have exhibited a larger scale in both spanwise and streamwise directions compared to the streamwise vortices in the undisturbed boundary layer and they contribute to the large-scale streamwise elongated velocity streaks in the near-wall region of the ramp, as shown in FIG. 29 (a). 


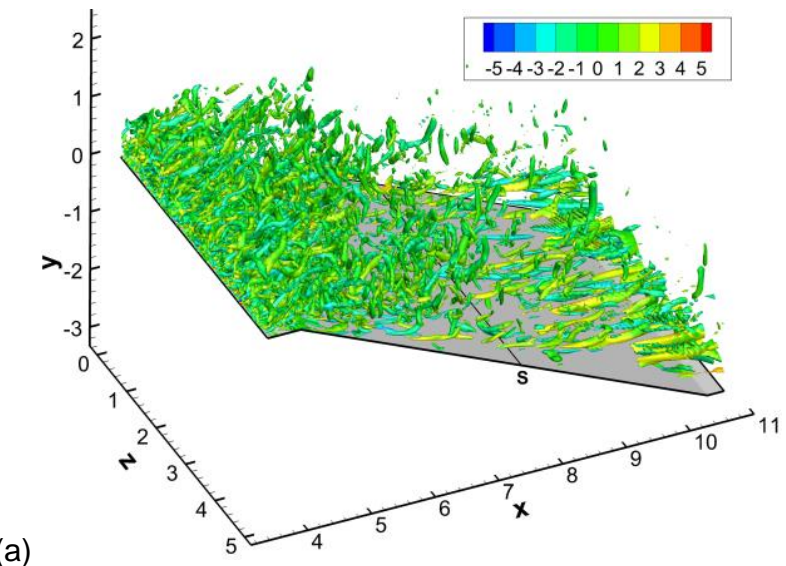

(a)

FIG. 31. Turbulence coherent structures in the ramp region visualized

(b)

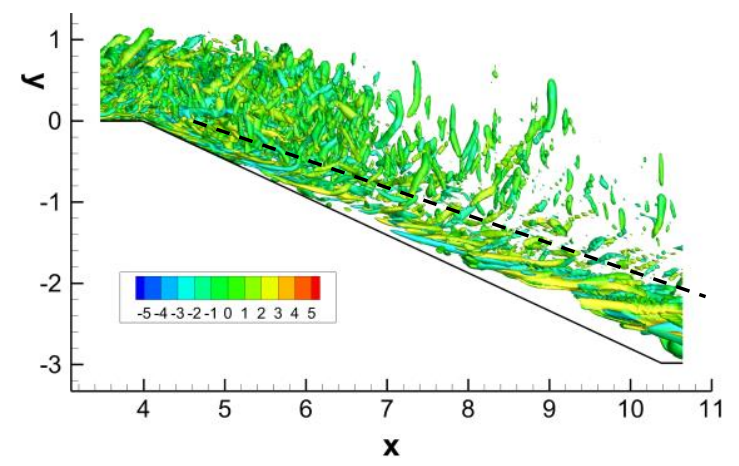
dash line in (b) marks the board between the inner and outer layers.

The iso-surface of the strength of the vorticity magnitude $\omega_{M}=\sqrt{\omega_{x}^{2}+\omega_{y}^{2}+\omega_{z}^{2}}$, is used to visualize turbulence vortex structures in FIG. 32. The large-scale streamwise tube-shape structures are clearly seen in the shear-layer around the separation region. These structures have a spanwise length scale of $\delta_{r e f}$ and a streamwise length scale of $5 \delta_{r e f}$, which provide other visual evidence of the existence of the large-scale Görtler vortices. After the flow reattachment, we can see the arch-shape structures in the mixing layer.

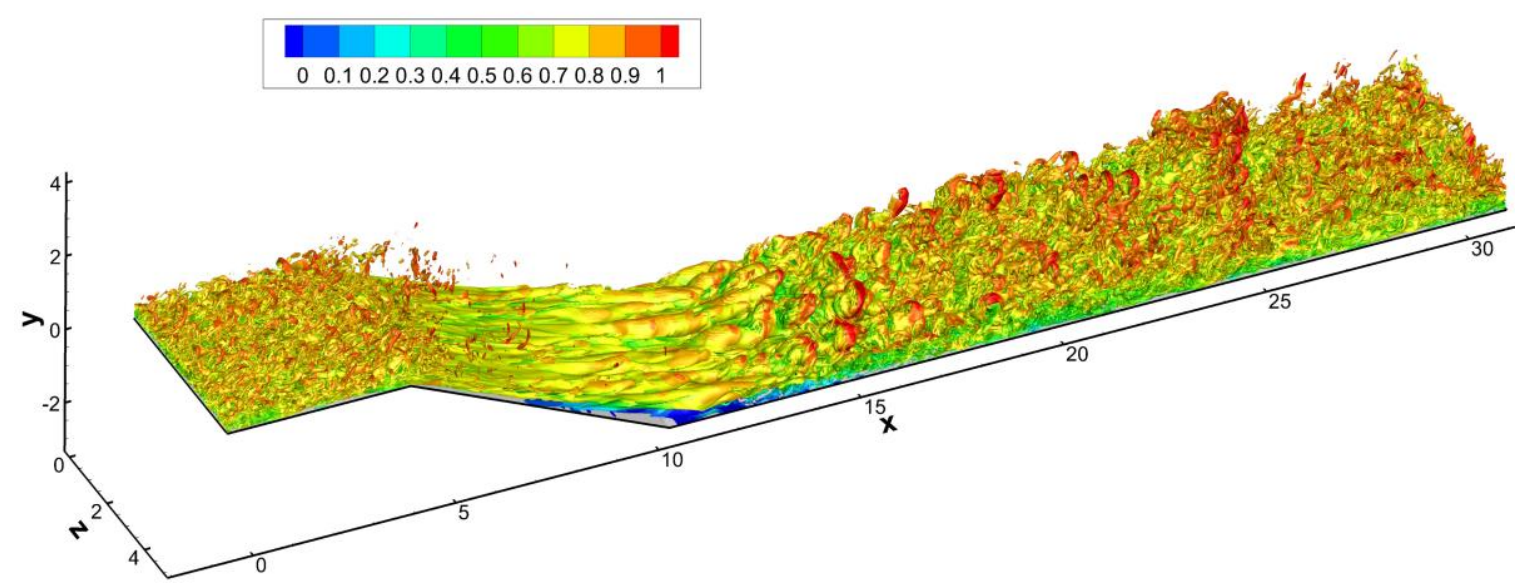

FIG. 32. Turbulence coherent structures visualized with the iso-surface of $\omega_{M}$ equalling to $1.5 \%$ of its global maximum and coloured with the $x$-velocity.

\section{CONCLUSIONS}

The Mach=2.9 supersonic turbulent flow over an expansion-compression corner with a $25^{\circ}$ incline angle at $\operatorname{Re}_{\delta}=$ 20000, 40000 and 80000 were studied using DNS. The results were well validated by comparing the wall properties, velocity profiles and turbulence intensities with published experimental measurements and other simulation data. Firstly, the statistics of wall properties (i.e. mean pressure distribution, pressure gradient, pressure fluctuation, skin friction, Stanton number and 
wall limiting streamlines) of the three cases were studied and the Reynolds number effects were also discussed. Detailed turbulence structures and flow statistics of the case $\mathrm{Re}_{\delta}=40000$ were then analysed.

It was found that with the increase of Reynolds number, the reattachment line moves upstream and the size of the separation bubble decreases. Despite that the skin friction and the heat flux increases are steeper after the flow reattachment for a higher Reynolds number case, the result of a lower Reynolds number case exhibits higher skin friction and peak wall heat flux in the recovery region.

In the ramp region, the turbulence motions and structures are first largely suppressed passing through the expansionwaves emitted from the expansion corner, and then amplified due to the interaction with the shock-wave formed at the compression corner region. During the expansion process, a two-layer turbulence structure is observed, in which the turbulence motions in the outer layer are suppressed over the entire ramp. In the inner layer, however, the turbulence motions are suppressed only in a small region around the expansion corners and then enhanced in the rest of the ramp. Therefore, a thin layer with a high level of turbulence fluctuations attaching to the edge of the separation bubble can be observed. Inside this thin layer, the quasi-streamwise vortices of the wall turbulence is well preserved. Downstream of the flow reattachment, another two-layer structure of turbulence, consisting of an outer decaying mixing layer and an inner regenerated wall turbulence, which is widely observed in SWTBLI flows, can also be identified in the present flow case.

The existence of large-scale Görtler-type vortices is visualized by the 3D wall streamlines around the reattachment line, where a typical saddle-node combination with a characteristic spanwise size of $\delta_{\text {ref }}$ can be seen. The Görtler vortices are responsible for the rapid increase of skin friction and high level of wall heat flux near the reattachment line. According to the analysis, the connection between the origin of the Görtler vortices and the well preserved large-scale streamwise vortices in the inner layer can be established, although the detailed relation between two requires further investigations, which is outside the scope of this study.

\section{ACKNOWLEDGMENTS}

This work is supported by the National Natural Science Foundation of China (11302012, 51420105008), the Leverhulme trust of UK (CE-12-10050002), the National Basic Research Program of China (2012CB720205), and the Astronautical Technology Innovation Foundation of China. Computer time for the present study was provided via the UK Turbulence Consortium (EPSRC grant EP/L000261/1) and the simulations were run on the UK High Performance Computing Service ARCHER. 


\section{REFERENCES}

1 D. S. Dolling, "Fifty years of shock-wave/boundary-layer interaction research: what next?” AIAA J. 39(8), 1517-1531 (2001).

2 D. D. Knight, H. Yan, A. G. Panaras, and A. A. Zheltovodov, "Advances in CFD prediction of shock wave turbulent boundary layer interactions," Prog. Aerosp. Sci. 39, 121-184 (2003).

3 A. A. Zheltovodov, "Some advances in research of shock wave turbulent boundary layer interactions," AIAA Paper No. 2006-0496, (2006).

4 D. D. Knight, and A. A. Zheltovodov, "Ideal-gas shock wave-turbulent boundary-layer interactions (STBLIs) in supersonic flows and their modeling: two-dimensional interactions," In: Shock wave-boundary-layer interactions, (Cambridge University Press, London, 2011) p. 137-201.

5 J. Sternberg, "The transition from a turbulent to laminar boundary layer," (Rep. No. 906, Ballistic Research Laboratory, Aberdeen Proving Ground, Maryland USA, 1954).

6 M. Morkovin, "Effects of high acceleration on a turbulent supersonic shear layer," Proceedings of the Heat Transfer and Fluid Mechanics Institute, Stanford University, (1955).

7 V. Zakkay, K. Toba, and T. Kuo, "Laminar transitional and turbulent heat transfer after a sharp convex corner," AIAA J. 2(8), $1389-1395(1964)$.

8 H. Thomann, "Effect of streamwise wall curvature on heat transfer in a turbulent boundary layer," J. Fluid Mech. 33, 383-392 (1968).

9 R. Page, and V. Sernas, "Apparent reverse transition in an expansion fan," AIAA J. 8(1), 189-190 (1970).

10 J. Lewis, R. Gran, and T. Kubota, "An experiment on the adiabatic compressible turbulent boundary layer in adverse and favourable pressure gradients," J. Fluid Mech. 51, 657-672 (1972).

11 R. Narasimpha, and P. K.Viswanath "Reverse transition at an expansion corner in supersonic flow", AIAA J. 13(5), 693695 (1975).

12 J. Dussauge, and J. Gaviglio, "The rapid expansion of a supersonic turbulent flow: role of bulk dilatation," J. Fluid Mech. 174, 81-1127 (1987).

13 M. A. Goldfeld, V. N. Zinov'ev, and V. A. Lebiga, "Structure and fluctuating characteristics of a compressible turbulent boundary layer behind an expansion fan," Fluid Dyn. 22, 40-45 (1987).

14 D. Smith, and A. Smits, "The rapid expansion of a turbulent boundary layer in a supersonic flow," Theor. Comput. Fluid 
Dyn. 2, 319-328 (1991).

15 J. Dawson, M. Samimy, and S. Arnette, "Effects of expansions on supersonic boundary layer: surface pressure measurements," AIAA J. 32(11), 2169-2177 (1994).

16 S. Arnette, M. Samimy, and G. Elliott, "The effects of expansion on the turbulence structure of a compressible boundary layer," J. Fluid Mech. 367, 67-105 (1998).

17 M. A. Goldfeld, R. V. Nestoulia, and A. N. Shiplyuk, "Re-laminarization of a turbulent boundary layer with a Mach number M=4”, J. Appl. Mech. Tech. Phys. 43(1), 76-82 (2002).

18 H. Thomann, "Effect of streamwise wall curvature on heat transfer in a turbulent boundary layer," J. Fluid Mech. 33, 383-392 (1968).

19 R. So, and G. Mellor, "Experiment on convex curvature effects in turbulent boundary layers," J. Fluid Mech. 60, 43-62 (1973).

20 J. Gillis, and J. Johnston, "Turbulent boundary-layer flow and structure on a convex wall and its redevelopment on a flat wall,” J. Fluid Mech. 135, 123-153 (1983).

21 A. Zheltovodov, L. Mecler, and E. Schülein, "Peculiarities of development of separated flows in compression corners after the expansion fans," Preprint No. 10-87, (Institute of theoretical and Applied Mechanics, USSR Academy of Sciences, Novosibirsk, 1987) p. 47 (in Russian).

22 A. Zheltovodov, and E. Schülein, "Peculiarities of turbulent separation development in disturbed boundary layers," Modelirovaniye v Mekchanike (Modelling in Mechanics), 2, 53-58 (1988).

23 A. A. Zheltovodov, E. G. Zaulichniy, V. M. Trofimov, and V. N. Yakovlev, "The study of heat transfer and turbulence in compressible separated flows," Preprint No. 22-87 (Institute of Theoretical and Applied Mechanics, USSR Academy of Sciences, Novosibirsk, 1987) (in Russian).

24 A. Zheltovodov, E. Schülein, and C. Horstman, "Development of separation in the region where a shock interacts with a turbulent boundary layer perturbed by rarefaction waves," J. Appl. Mech. Tech. Phys. 34(3), 346-354 (1993),

25 A. V. Borisov, A. A. Zheltovodov, V. M. Trofimov, E. Schülein, and V. N Yakovlev, "Analysis of turbulent separated flows in the vicinity of two-dimensional obstacles under supersonic speeds conditions," Proceedings of separated flows and jets IUTAM symposium Novosibirsk/USSR 1990, edited by V.V. Kozlov, and A.V. Dovgal (Springer-Verlag Berlin Heidelberg 1991), p. 333-336.

26 A. Zheltovodov, A. Borisov, D. Knight, C. Horstman, and G. Settles, "The possibilities of numerical simulation of shock 
waves/boundary layer interaction in supersonic and hypersonic flows," Proceedings of international conference on methods of aerophysical research (Russian Academy of Sciences, Siberian Division, Novosibirsk, 1992) p. 164-170.

27 layer interactions," Preprint No. 2-93, (Institute of Theoretical and Applied Mechanics RAS, Novosibirsk, 1993).

28 interaction," Proceedings: International conference on methods of aerophysical research, Institute of theoretical and Applied Mechanics, Russian Academy of Sciences, Novosibirsk, Russia, 2, 118-125 (1994).

29 578 (1998). corner," Proceedings of the third AFOSR international conference on DNS/LES, edited by C. Liu, L. Sakkel, and T. Beutner. (Greydon Press, Columbus, Ohio, USA, 2001), p. 183-194. under the conditions of supersonic separated flows," Abstracts: IUTAM Symposium on Separated Flows and Jets. USSR Academy of Sciences, Siberian Division, Novosibirsk, USSR, p. 273-274 (1990). Comut. Fluid Dyn. 25(7), 407-423 (2011).

33 1935-1944 (2002). high-speed turbulent flows,” J. Sci. Comput. 56, 67-95 (2013).

35 Phys. 136, 83-99 (1997). simulation of compressible turbulent flows," Comput. Fluids 104, 55-72 (2014). 
39 N. D. Sandham, Q. Li, and H. C. Yee, "Entropy splitting for high-order numerical simulation of compressible turbulence," J. Comput. Phys. 178, 307-322 (2002).

40 S. Gottlieb, and C. W. Shu, "Total variation diminishing Runge-Kutta schemes," Math. Comput. 67, 73-85 (1998).

41 P. D. Thomas, and C. K. Lombard, "Geometric conservation law and its application to flow computations on moving grids," AIAA J. 17(10), 1030-1037 (1979).

42 E. Touber, and N. D. Sandham, "Large-eddy simulation of low-frequency unsteadiness in a turbulent shock-induced separation bubble," Theor. Comput. Fluid Dyn. 23(2), 79-107 (2009).

43 E. Touber, and N. D. Sandham, "Oblique shock impinging on a turbulent boundary layer: low-frequency mechanisms," AIAA Paper No. 2008-4170, (2008).

44 T. S. Lund, X. Wu, and K. D. Squires, "Generation of turbulent inflow data for spatially-developing boundary layer simulations," J. Comput. Phys. 140, 233-258 (1998).

45 P. Sagaut, E. Garnier, E. Tromeur, L. Larcheveque, and E. Labourasse, "Turbulent inflow conditions for large--eddy simulation of compressible wall-bounded flows," AIAA J. 32(3), 469-477 (2004).

46 B. Morgan, J. Larsson, S. Kawai, and S. K. Lele, "Improving low-frequency characteristics of recycling/rescaling inflow turbulence generation," AIAA J. 49(3), 582-597 (2011).

47 X. Wu, and P. Moin, "Direct numerical simulation of turbulence in a nominally zero-pressure-gradient flat-plate boundary layer," J. Fluid Mech. 630, 5-41 (2009).

48 J. W. Kim, and D. J. Lee, "Generalized characteristic boundary conditions for computational aeroacoustics," AIAA J. 38(11), 2040-2049 (2000).

49 J. W. Kim, and D. J. Lee, "Generalized characteristic boundary conditions for computational aeroacoustics, part 2," AIAA J. 41(1), 47-55 (2004).

50 P. Sagaut, "Theoretical background: large-eddy simulation," Large-eddy simulation for acoustics, (Cambridge University Press, Cambridge, 2007), 89-127.

51 M. S. Loginov, N. A. Adams, and A. A. Zheltovodov "Large eddy simulation of shock- wave/turbulent-boundary-layer interaction," J. Fluid Mech. 565(1), 135-169 (2006).

52 X. Gloerfelt, and P. Lafon, "Direct computation of the noise induced by a turbulent flow through a diaphragm in a duct at low Mach number," Comput. Fluids 37, 388-401 (2008).

53 F. Gao, G. Zambonini, J. Boudet, X. Ottavy, L. Lu, and L. Shao, "Unsteady behavior of corner separation in a 
compressor cascade: Large eddy simulation and experimental study", J. Power and Energy, 229(5), 508-519 (2015).

J. Murlis, H. M. Tsai, and P. Bradshaw, "The structure of turbulent boundary layers at low Reynolds numbers," J. Fluid Mech. 122, 12-56 (1982).

55 L. P. Erm, and P. N. Joubert, "Low Reynolds number turbulent boundary layers," J. Fluid Mech. 230, 1-44 (1991).

56 L. P. Purtell, P. S. Klebanoff, and F. T. Buckley, "Turbulent boundary layer at low Reynolds number," Phys. Fluids. 24, 802-811 (1981).

57 P. R. Spalart, "Direct numerical simulation of a turbulent boundary layer up to $\operatorname{Re}_{\theta}=1410$," J. Fluid Mech. 187, 61-98 (1988).

S. Pirozzoli, M. Bernardini, and F. Grasso, "Direct numerical simulation of transonic shock/boundary layer interaction under conditions of incipient separation," J. Fluid Mech. 657, 361-393 (2010).

59 M. Wu, "Direct Numerical Simulation of Shockwave and Turbulent Boundary Layer Interactions," PhD Thesis, Princeton University, Princeton, 2007.

60 S. Priebe, M. Wu and M. P. Martín, "Direct numerical simulation of a reflected-shock-wave/turbulent-boundary-layer interaction,” AIAA J. 47(5), 1173-1185, (2009).

61 A. Roshko, and G. J. Thomke, "Observations of turbulent reattachment behind an axisymmetric downstream-facing step in supersonic flow," AIAA J. 4(6), 975-980 (1966).

62 H. Görtler, "On the three-dimensional instability of laminar boundary layers on concave walls," (NASA Tech. Report, No. 19780078801, 1954).

63 J. J. Ginoux, "Experimental evidence of three-dimensional perturbations in the reattachment of a two-dimensional laminar boundary layer at M =2.05," (Tech. Rep. TCEA TN1, Training Center for Experimental Aerodynamics, RhodeSaint-Genese, Belgium, 1958).

64 J. M. Floryan, “On the Görtler instability of boundary layers,” Prog. Aerosp. Sci. 28, 235-271 (1991).

65 I. Tani, "Production of longitudinal vortices in the boundary layer along a concave wall," J. Geophys. Res. 67(8), 30753080 (1962).

66 A. J. Smits, S. T. B. Young, and P. Bradshaw, "The effect of short regions of high surface curvature on turbulence boundary layers," J. Fluid Mech. 94, 209-242 (1979).

${ }^{67}$ M. S. Selig, J. Andreopoulos, K. C. Muck, J. P. Dussauge, and A. J. Smits, "Turbulent structure in a shock wave/turbulent boundary-layer interaction," AIAA J. 27(7), 862-869 (1989). 
68 A. A. Zheltovodov, E. Schülein, and V. N. Yakovlev, "Development of turbulent boundary layer under conditions of mixed interaction with shock and expansion fans," (Preprint 28-83, Institute of Theoretical and Applied Mechanics, USSR Academy of Sciences, Novosibirsk, 1983) (in Russian).

69 E. Schülein, and V. V. Trofimov, "Steady longitudinal vortices in turbulent separated flows,” J. Fluid Mech. 672, 451$476(2011)$.

70 J. R. Edwards, "Numerical simulations of shock/boundary layer interactions using time-dependent modeling techniques -a survey of recent results,” Prog. Aerosp. Sci. 44, 447-465 (2008).

71 T. Sayadi, C. W. Hamman, and P. Moin, "Direct numerical simulation of complete H-type and K-type transitions with implications for the dynamics of turbulent boundary layers,” J. Fluid Mech. 724, 480-509 (2013).

72 S. Pirozzoli, and F. Grasso, "Direct numerical simulation of impinging shock wave/turbulent boundary layer interaction at M=2.25," Phys. Fluids 18, 065113 (2006).

73 A. Jammalamadaka, Z. Li and F. Jaberi, "Numerical investigations of shock wave interactions with a supersonic turbulent boundary layer,” Phys. Fluids 26, 056101 (2014).

74 J. Kim, P. Moin, and R. Moser, "Turbulence statistics in fully developed channel flow at low Reynolds number," J. Fluid Mech. 177, 133-166 (1987).

75 S. Pirozzoli, and M. Bernardini, "Direct numerical simulation database for impinging shock wave/turbulent boundarylayer interaction," AIAA J. 49(6), 1307-1312 (2011).

76 J. Zhou, R. J. Adrian, S. Balachandar, and T. M. Kendall, "Mechanisms for generating coherent packets of hairpin vortices in channel flow," J. Fluid Mech. 387, 353-396 (1999). 